Montaser Bakroon, Reza Daryaei, Daniel Aubram, Frank Rackwitz

\title{
Investigation of Mesh Improvement in Multimaterial ALE Formulations Using Geotechnical Benchmark Problems
}

Journal article | Accepted manuscript (Postprint)

This version is available at https://doi.org/10.14279/depositonce-10470

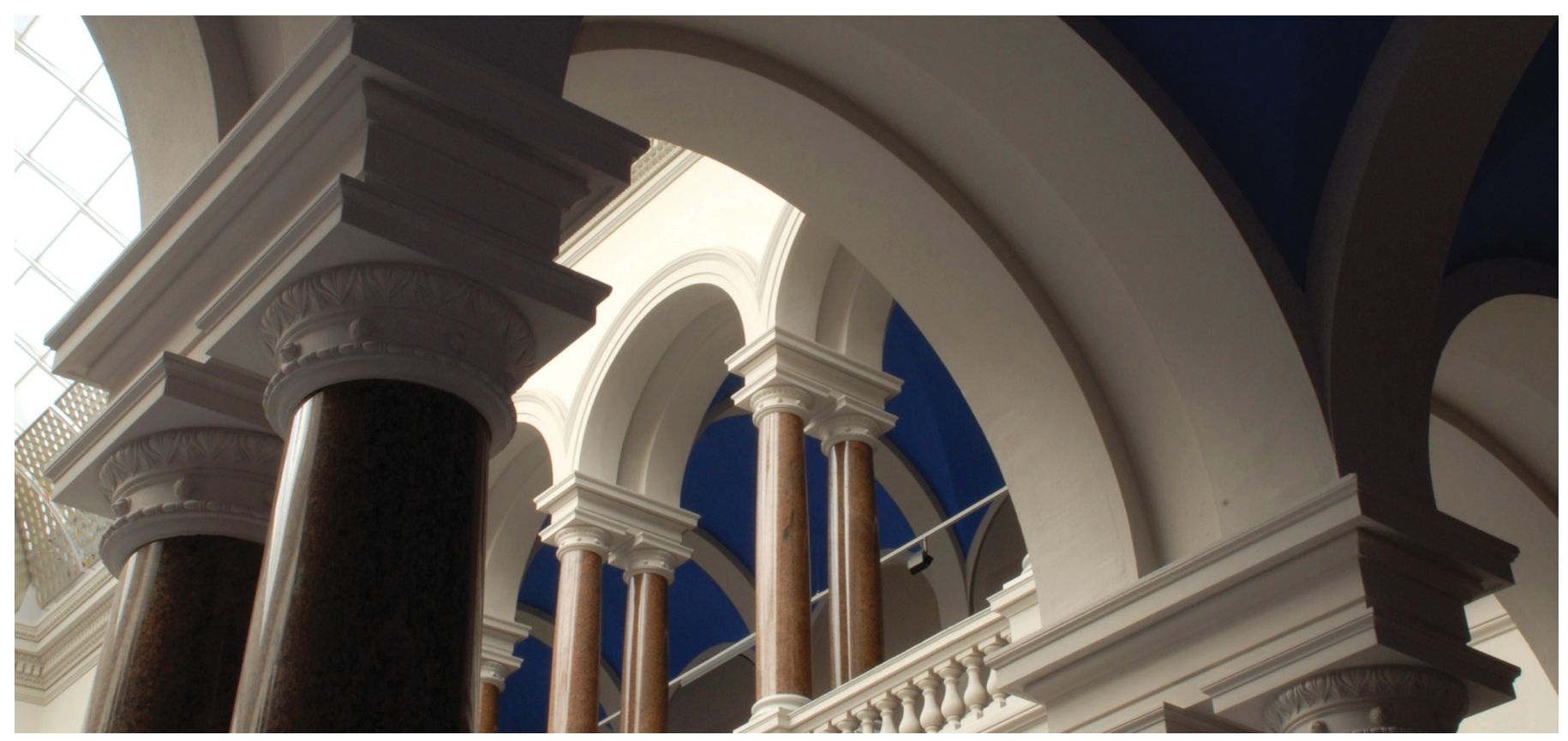

This material may be downloaded for personal use only. Any other use requires prior permission of the American Society of Civil Engineers. This material may be found at https://doi.org/10.1061/(asce) gm.1943-5622.0001723

Bakroon, M., Daryaei, R., Aubram, D., \& Rackwitz, F. (2020). Investigation of Mesh Improvement in Multimaterial ALE Formulations Using Geotechnical Benchmark Problems. International Journal of Geomechanics, 20(8), 04020114. https://doi.org/10.1061/(asce)gm.1943-5622.0001723 


\section{Investigation of mesh improvement in multi-material ALE formulations us-}

2 ing geotechnical benchmark problems

3 Montaser Bakroon ${ }^{1}$ (Corresponding author)

4 Reza Daryaei ${ }^{2}$

5 Daniel Aubram ${ }^{3}$

$6 \quad$ Frank Rackwitz

$7 \quad{ }^{1}$ Research Scholar, Chair of Soil Mechanics and Geotechnical Engineering, Technische Universität Berlin,

8 TIB1-B7, Gustav-Meyer-Allee 25, 13355, Berlin, Germany

$9{ }^{2}$ Research Scholar, Chair of Soil Mechanics and Geotechnical Engineering, Technische Universität Berlin,

10 TIB1-B7, Gustav-Meyer-Allee 25, 13355, Berlin, Germany

$11{ }^{3}$ Senior Research Associate, Chair of Soil Mechanics and Geotechnical Engineering, Technische Universität

12 Berlin, TIB1-B7, Gustav-Meyer-Allee 25, 13355, Berlin, Germany

$13{ }^{4}$ Professor, Head of Chair of Soil Mechanics and Geotechnical Engineering, Technische Universität Berlin,

14 TIB1-B7, Gustav-Meyer-Allee 25, 13355, Berlin, Germany

15 Abstract

16 Two of the mesh-based numerical approaches suitable for geotechnical large deformation problems, the multi-

17 material ALE (MMALE) and the Coupled Eulerian-Lagrangian (CEL) methods are investigated. The remeshing step in MMALE is claimed to hold advantages over CEL, but its effects on application problems are not studied in detail. Hence, the possible capabilities and improvements of this step are studied in three large deformation geotechnical problems with soil-structure interaction. The problems are validated and verified using experimental and analytical solutions, respectively. By using the remeshing step in MMALE, a smoother material interface, lower remap-related errors, and better computation cost are achieved.

\section{Keywords}

Multi-Material Arbitrary Lagrangian Eulerian, Coupled Eulerian-Lagrangian, large deformations, remeshing, interface reconstruction

\section{Introduction}

Small deformation geotechnical problems can be adequately analyzed by using conventional

28 Lagrangian FEM. However, such an approach exhibits considerable shortcomings when the soil 29 undergoes significant deformation. Examples include pile penetration, soil cutting, slope failures, and

30 liquefaction events. Hence, efforts were made to develop methods that simulate the numerical problems

31 associated with large material deformation. 
There are various methods to handle such numerical problems which can be categorized into two classes, point-based and mesh-based methods (here only methods derived from continuum mechanics assumption are considered). Examples of point-based methods are material point method (MPM) (Bardenhagen et al., 2000) and smoothed particle hydrodynamics (SPH) (Gingold and Monaghan, 1977), whereas classical FEM (small-strain Lagrangian), Eulerian, ALE, and CEL methods are listed as mesh-based methods (Aubram et al., 2015). Concerning methods that rely on a computational mesh, the most promising approaches include the Coupled Eulerian-Lagrangian (CEL) method and the Arbitrary Lagrangian-Eulerian (ALE) method, which is chosen for this study. The latter can be subdivided into Simplified ALE (SALE) and Multi-Material ALE (MMALE) methods. These methods are popular in fluid dynamics yet not well-known and extensively used in the context of geomechanics. Therefore, the motivation of this paper is to evaluate the possible advantages of MMALE over CEL in case of large deformation geotechnical problems.

Two categories of ALE are generally distinguished, based on a number of materials that might be present in a single element (Fig. 1). Simplified ALE (SALE) approaches resolve material boundaries (free surfaces or material interfaces) in a Lagrangian way using edges and faces (in 3D) of the computational mesh. Therefore, each mesh element is filled with only one material. Unlike SALE, MMALE allows multiple materials to be defined in each element such that material boundaries can flow through the mesh. This method reconstructs the interfaces between multiple materials, making it is suitable to model more complicated and large deforming problem. Fig. 1 provides a schematic comparing all the methods discussed in the present study.

There are various applications of CEL in literature concerned with large deformation problems in geomechanics and geotechnical engineering, e.g., (Bakroon et al., 2019; Heins and Grabe, 2017). One of the earliest works is that done by Qiu et al. (2011), where three numerical benchmarks were used to assess CEL. It was argued that CEL is well suited for large geotechnical problems. Similar conclusions were drawn in a comprehensive and thorough study conducted by Wang et al. (2015) concerning three different numerical approaches, including CEL.

Concurrent to CEL studies, several works were done in applying the ALE method to geotechnical 
is the "remeshing and interpolation technique with small strain", RITSS method developed by Hu and

61 Randolph (1998a). In this method, after 10-20 steps of simple infinitesimal strain incremental analysis a rezoning step is performed. Since then, this method is subjected to many improvements and applications such as inclusion of an h-adaptivity rezoning (Hu and Randolph, 1998b) which is then used to simulate pullout test (Song et al., 2008). Similarly, in a series of works done at the university of Newcastle for instance by Nazem et al. (2008) and Sabetamal et al. (2014), an ALE method with coupled formulation was developed to simulate problems such as offshore large deformation problems. In a work done by Aubram et al. (2015), an advanced SALE formulation is implemented, and its performance is evaluated by simulating shallow and pile penetration into the sand. A good agreement between numerical results and experimental measurements was observed.

On the other hand, Bakroon et al. (2018) assessed the feasibility of SALE in large geotechnical deformation problems. It was concluded that for extremely large problems, the SALE exhibits shortcomings, unlike MMALE which converged to a solution. Therefore, MMALE was suggested to be considered as an alternative approach to SALE for solving complex large deformation problems. Consequently, studies focused on applying the MMALE to geotechnical problems.

The structure of this study is as follows. In Section 0, details of the numerical implementation of CEL and MMALE algorithms such as operator splitting, remeshing, and remapping steps, and soil-structure coupling are described. Section 0 presents three numerical examples to investigate the performance of CEL and MMALE, including a discussion of the results. Concluding remarks are provided in Section 0.

\section{Details of MMALE and CEL}

80 The original CEL method was developed by Noh (1964). In this method, the material regions are treated as Eulerian, while the region boundaries are defined as polygons which are then approximated by Lagrangian meshes overlapping the Eulerian mesh. The Eulerian mesh is fixed throughout the analysis. Some commercial codes implemented variants of the original CEL approach. In the particular CEL method used in this study, a Lagrangian step is first conducted which solves the physics of the problem by using a mesh which deforms with the material. In the case of the pure Lagrangian as well as the Lagrangian step in SALE, MMALE, and CEL, employed in this work, the updated Lagrangian (UL) 
87 (Belytschko et al., 2000; Hallquist, 2006) is used. Concerning the utilized objective stress rate, the 88 Jaumann rate is used (Hallquist, 2006; Livermore Software Technology Corporation, 2015).

89 After performing the Lagrangian step, the mesh is rezoned to its initial configuration to maintain mesh quality (rezoning/remeshing step). Subsequently, the solution is transported from the deformed mesh to the updated/original mesh (remapping/advection step). This method is different than the CEL method developed by Noh (1964) where the Eulerian solution is not divided into a rezone and remap step

93 (Benson, 1992).

94 The Arbitrary Lagrangian-Eulerian (ALE) method has been developed by Hirt et al. (1974) and Trulio and Trigger (1961) to address the mesh distortion issue attributed to classical Lagrangian approaches. In each ALE calculation cycle, similar to CEL, the general strategy is to perform a three-step scheme consisting of a Lagrangian step, a remeshing (rezone) step, and a remapping step. After the Lagrangian step, the rezone step relocates the nodes of the mesh in such a way that mesh distortion is reduced. Unlike CEL, however, the updated mesh is not necessarily identical to the original mesh but could be

100 obtained through the application of a smoothing algorithm (Donea et al., 2004). Finally, the remapping

101 step transfers the solution variables from the old onto the new (rezoned) mesh.

102 The focus of this paper is to evaluate the remeshing step in MMALE and CEL as the main distinguishing 103 factor between these methods. The general solving strategy has been discussed in section 0 , which is also available in the literature (Benson, 1992).

105 Therefore, the remeshing step, as well as some other features of MMALE and CEL, are described in 106 this section.

\section{Operator splitting}

108 Generally spoken, operator splitting is a strategy to divide a complicated equation into a sequence of 109 simpler equations (Benson, 1992). Operator splitting can be used to solve the general Eulerian 110 conservation equation:

$$
\frac{\partial \phi}{\partial t}+\boldsymbol{\nabla} \cdot \boldsymbol{\Phi}=\boldsymbol{S}
$$

111 Where $\phi$ is the field variable, $\boldsymbol{\Phi}$ is the flux function, and $\boldsymbol{s}$ is the source term. This equation can be solved 112 whether in one step (Bayoumi and Gadala, 2004; Donea et al., 1982) or alternatively in multiple steps 
113 where the equation is broken up into a series of less complicated equations, i.e., into a Lagrangian term

$114\left(\frac{\partial \phi}{\partial t}=\boldsymbol{S}\right)$ and a Eulerian term $\left(\frac{\partial \phi}{\partial t}+\boldsymbol{\nabla} \cdot \boldsymbol{\Phi}=0\right)$ (Benson, 1992). The schematic view of operator splitting is 115 drawn in Fig. 2.

\section{Remeshing step (Mesh smoothing algorithms)}

117 The main difference between CEL and ALE (SALE and MMALE) emerges when one compares the 118 remeshing (rezoning) step in both methods. In case of remeshing step in CEL, the new mesh is trivially 119 the original mesh at the beginning of the calculation, while in ALE, the remeshing step is performed by 120 using mesh smoothing algorithms that produce a new, less distorted mesh based on the deformed mesh 121 of the Lagrangian step. The new mesh is not necessarily the original mesh of CEL.

122 To define a robust rezoning algorithm, two criteria must be satisfied. First, the quality of the grid 123 elements must be maintained. Second, the grid should be focused on zones with a rapid variation of 124 material flow to reduce computational errors, which is referred to as the adaptivity control criterion.

125 While these goals seem easy to achieve, they expose a challenge in the derivation of a robust rezoning 126 algorithm. If one considers quality maintenance as the only important factor, then accuracy in areas of 127 high variations will be lost, since pretty similar sizes will be assigned to rezoned grid elements. 128 Algorithms developed merely on this criterion may be strongly dependent on mesh quality, which may not provide a unique solution. Weighting each criterion is therefore difficult, and it may be problem

130 dependent (Knupp et al., 2002).

131 Rezoning/smoothing techniques can either change the nodal connectivity, such as h-adaptivity where 132 new elements are generated, or keep the nodal connectivity and only relocate the nodes such as r133 adaptivity method where the node position are relocated to obtain a smoother mesh (Di et al., 2007).

134 The focus here is to study those smoothing methods where the nodal connectivities are not changed.

135 Such rezoning algorithms can be divided into different groups, each having its advantages and 136 drawbacks. Coordinate- or grid-based algorithms can be applied to the gird locally or globally. In local 137 coordinate-based algorithms, the nodes are moved based on local criteria (Benson, 1989; Donea et al., 138 1982). For example, based on neighboring element areas around the node, a ratio of minimum to the 139 maximum area as well as the maximum cosine value of the vertex angles connecting this node to other 
140 nodes is calculated. By these two values, the movement requirement of the node will be determined

141 (Benson, 1989). The shortcoming of this method is that it is based on ad hoc quality measures, which

142 means this class of problems is only applicable to a specific group of problems. In addition, there is no

143 guarantee that the resulting mesh is unfolded (Knupp et al., 2002).

144 An example of a global smoothing algorithm is the one developed by Brackbill and Saltzman (1982), 145 where they modified the Winslow algorithm (Winslow, 1967). Extra terms were added to make the 146 smoothing algorithm stronger. However, the coefficients of such terms are assigned somewhat arbitrary 147 and without a clear guide. In addition, this method is independent of the Lagrangian grid, which makes 148 the resulting mesh, far from the Lagrangian mesh. To resolve this issue, an iterative approximate 149 solution is used. However, it is not guaranteed if the resulting grid is unfolded. Besides, there is no 150 theory to specify the number of iterations by the user (Knupp et al., 2002).

151 There are numerous studies in remeshing techniques, but to the knowledge of the authors, this step is 152 the least developed aspect of ALE methods. A short description of the three popular methods will be 153 provided.

\section{$154 \quad$ Volume-weighted smoothing}

155 To better clarify the smoothing methods, Fig. 3 was drawn where the arbitrary node K, is supposed to 156 be rezoned (relocated). Variables subscripted with Greek letters refer to element variables while 157 subscripts with capital letters refer to local node numbering within an element. Also, the letter A is an 158 arbitrary letter corresponding to the nodes of each element adjacent to node K. Therefore in case of the 159 2D mesh in Fig. 3, A can be $L$ or $E$, or $K$.

160 In volume weighted smoothing, the new position of the node is determined by using the volume of each 161 neighboring element sharing that node. The method is illustrated by Eq. (2) and (3).

162 First, the nodal coordinates of each element adjacent to node K, $\vec{x}_{A}$ are averaged using (2) to obtain the 163 coordinate $\vec{x}_{\alpha}$ (the point is marked with red cross in Fig. 3). The parameter, $N$, corresponds to numbers 164 of element nodes, which can be four or eight for two- and three dimensions, respectively. 
165 The new position of the node $\mathrm{K}, \vec{x}_{K}^{*}$, is then obtained by the volume-weighted averaging as in Eq. (3)

166 using the volume of each adjacent element, $V_{\alpha}$, and the total number of adjacent elements, $n_{\text {adj }}$ (Ghosh

167 and Kikuchi, 1991):

$$
\begin{gathered}
\vec{x}_{\alpha}=\frac{1}{N} \sum_{A=1}^{N} \vec{x}_{A} \\
\vec{x}_{K}^{*}=\frac{\sum_{\alpha=1}^{n_{a d j}} V_{\alpha} \vec{x}_{\alpha}^{n}}{\sum_{\alpha=1}^{n_{a d j}} V_{\alpha}}
\end{gathered}
$$

168

\section{Equipotential smoothing}

This method is more complicated than the previous methods and is intended to smooth the whole mesh or a part of it globally. The equipotential method is based on the solution of the Laplace equation (5) associated with the logical, generally curvilinear coordinates representing the grid lines in structured meshes (Winslow, 1963). The concept is to solve (5) for the Cartesian coordinates of the mesh lines, that is $\mathrm{x}\left(\xi_{i}\right),(i=1,2,3)$ instead of the curvilinear coordinates $\xi=\left(\xi_{1}, \xi_{2}, \xi_{3}\right)$, resulting in Eq. (6). In this method, all the element faces which share the node $\mathrm{K}$ are considered in the calculation (Nodes $\mathrm{L}$ and $\mathrm{E}$ in Fig. 3). Therefore, in two dimensions, eight nodes will be studied while in three dimensions, eighteen nodes will be studied (Fig. 3). For more information regarding the calculation process, the reader is advised to see the work done by Souli et al. (2000).

$$
\begin{gathered}
\nabla^{2} \xi=0 \\
\gamma_{1} \partial_{\xi_{1} \xi_{1}} \mathbf{x}+\gamma_{2} \partial_{\xi_{2} \xi_{2}} \mathbf{x}+\gamma_{3} \partial_{\xi_{3} \xi_{3}} \mathbf{x}+2 \beta_{1} \partial_{\xi_{1} \xi_{2}} \mathbf{x}+2 \beta_{2} \partial_{\xi_{1} \xi_{3}} \mathbf{x}+2 \beta_{3} \partial_{\xi_{2} \xi_{3}} \mathbf{x}=0
\end{gathered}
$$

183 where 


$$
\begin{gathered}
\gamma_{i}=\partial_{\xi_{i}} x_{1}^{2}+\partial_{\xi_{i}} x_{2}^{2}+\partial_{\xi_{i}} x_{3}^{2} i=1,2,3 \\
\beta_{1}=\left(\partial_{\xi_{1}} \mathbf{x} \cdot \partial_{\xi_{3}} \mathbf{x}\right)\left(\partial_{\xi_{2}} \mathbf{x} \cdot \partial_{\xi_{3}} \mathbf{x}\right)-\left(\partial_{\xi_{1}} \mathbf{x} \cdot \partial_{\xi_{2}} \mathbf{x}\right) \partial_{\xi_{3}} \mathbf{x}^{2} \\
\beta_{2}=\left(\partial_{\xi_{2}} \mathbf{x} \cdot \partial_{\xi_{1}} \mathbf{x}\right)\left(\partial_{\xi_{3}} \mathbf{x} \cdot \partial_{\xi_{1}} \mathbf{x}\right)-\left(\partial_{\xi_{2}} \mathbf{x} \cdot \partial_{\xi_{3}} \mathbf{x}\right) \partial_{\xi_{1}} \mathbf{x}^{2} \\
\beta_{3}=\left(\partial_{\xi_{3}} \mathbf{x} \cdot \partial_{\xi_{2}} \mathbf{x}\right)\left(\partial_{\xi_{1}} \mathbf{x} \cdot \partial_{\xi_{2}} \mathbf{x}\right)-\left(\partial_{\xi_{3}} \mathbf{x} \cdot \partial_{\xi_{1}} \mathbf{x}\right) \partial_{\xi_{2}} \mathbf{x}^{2}
\end{gathered}
$$

184 To investigate quantitatively the effectiveness of each smoothing method, a simple numerical model

185 was developed, as shown in Fig. 4. The model consists of nine elements where the upper right node is

186 subjected to a displacement in both horizontal and vertical directions. The left lateral and the lower edge

187 of the model is fixed. An elastic material model is assumed. After displacement, the deformed mesh is

188 evaluated based on the so-called Jacobian distortion index ranging from 0 to 1 . This index describes the

189 deviation of the element from its ideal rectangular form. A value close to 1 indicates an element whose

190 shape is close to its ideal form, while a value of 0 indicates a heavily distorted element (Plaxico et al.,

191 2009). In Fig. 4 the distortion index is shown in percentage.

192 Without using any smoothing method, representing a purely Lagrangian mesh, the deformation is

193 significant in the upper right element and its three adjacent elements. On the other hand, by using the

194 smoothing methods, the distortion is decreased. In this simple example, all smoothing methods provided acceptable results. Another model was also developed where further displacement was applied. In the upper right element, a non-convex element was obtained, and none of the smoothing methods could handle the non-convex element and provided a folded mesh.

198 Indeed, the present example is too simple to study the performance of each smoothing method 199 thoroughly. The smoothing methods will be later discussed using a benchmark model in section 0 .

\section{$200 \quad$ Remapping step}

201 After generating a new grid, the solution variables have to be transferred to the new mesh. There are several methods to remap the solution from the Lagrangian mesh onto the new mesh (Benson, 1992; Margolin and Shashkov, 2003). Because the mesh topology does not change in both ALE and Eulerian methods, the remap can be stated as an advection problem which can be solved using conservative finite difference or finite volume methods. In such advection algorithms, the difference between the reference and the rezoned grid is interpreted as volume flux, that is, the change of element/cell volume equals the 
sum of in- and outfluxes across the cell boundary. The updated value of cell-centered solution variables

208 is then determined by calculating the influx and outflux of this variable in each cell using the

209 information of the adjacent cells. Conventionally, each advection algorithm is applied in one coordinate

210 direction and then extended to two or three dimensions using the operator-split technique (Benson 1992;

211 Souli and Benson, 2013).

212 Another group of remapping algorithms treats the intersection of the reference and rezoned grid as

213 polygons or polyhedra (Berndt et al., 2011; Kucharik and Shashkov, 2012; Margolin and Shashkov,

214 2003). One of the main differences between these two concepts is the way to treat mixed/multi-material

215 cells. When using advection algorithms, the mixed cells are treated differently than the pure cell, while

216 in intersection-based remapping, both pure and mixed cells are treated alike. For more information

217 about the remapping method based on polygons and polyhedra, the reader is referred to (Berndt et al.,

218 2011; Chazelle, 1989, 1994; Kucharik and Shashkov, 2012; Margolin and Shashkov, 2003).

219 The current remapping algorithms used in geotechnical engineering are mostly based on advection

220 algorithms. A more detailed description regarding the most utilized advection algorithms, namely the

221 first-order accurate donor cell and second-order accurate Van Leer (MUSCLE) scheme is available the

222 literature (Benson, 1992).

\section{Soil-structure coupling}

224 Almost all problems in geotechnical engineering are characterized by soil-structure-interaction and contact between different materials. Multi-material elements in CEL or MMALE naturally handle contact without contact elements or algorithms (Benson and Okazawa, 2004). These elements use the same velocity for all materials, which is a manifestation of the "no slip" contact condition in mixture theory. However, in many soil-structure-interaction problems, like pile penetration, interfacial slip, and frictional contact play an important role. Moreover, in many situations, the soil undergoes large

230 deformations while deformation of the structure is moderate. Coupling between Lagrangian and non-

231 Lagrangian parts becomes necessary in such cases.

232 A penalty contact scheme is utilized in most codes owing to its simplicity and robustness. As a simple description, the penalty method applies springs between nodes of Lagrangian and the Eulerian parts. These springs have seeds and anchors. The seeds are attached to the Lagrangian nodes, while anchors 
are attached to the Eulerian nodes. In practice, it is better to have more nodes in the Lagrangian part

236 interface, to ensure that at least one Eulerian node is tracked by one Lagrangian node. The spring forces

237 are calculated based on the relative penetration of master and slave parts, and the calculated contact

238 spring stiffness.

\section{Numerical Examples}

240 In this section, three application problems are presented which exhibit specific challenges in numerical

241 simulation. Such classical examples are crucial for comparison of different numerical methods since

242 they have a reduced number of complexities. These examples are modeled using MMALE and CEL,

243 and the corresponding results are compared. The comparison includes the calculation time, and the

244 effect of mesh density on it, accuracy in terms of leakage, interface, and energy loss, which will be

245 described during the section. Table 1 lists the comparison criteria and their specific purpose for each

246 numerical example discussed in this section.

247 For all simulations mentioned in this study, the calculations were carried out in the commercial code,

248 LS-DYNA ${ }^{\circledR}$, on a server with two $2.93 \mathrm{GHz}$ quad-core Intel CPU X5570 processors and $48 \mathrm{~GB}$ of RAM.

249 A short description of the element technology and time stepping is provided for completeness. For

250 SALE, 1-point ALE elements are used while for MMALE and CEL, 1-point reduced integration

251 elements are used. Among the various smoothing methods, equipotential smoothing for the MMALE

252 simulations is applied. This smoothing algorithm is commonly used and provides more stable results

253 compared to other methods. For the advection step, van Leer method is chosen over donor cell since it

254 benefits from second-order accuracy (Benson, 1992).

255 Most CEL and ALE methods use explicit schemes to advance the solution in time. In explicit methods,

256 to maintain stability and acceptable accuracy, an appropriate time step size must be assigned. The

257 critical time step can be estimated by

$$
\Delta t_{e}=\frac{L_{s}}{c}
$$

258 where $L_{s}$ is the characteristic length of the element, and $c$ is the sound speed in the corresponding

259 material. Determining a suitable time step size is crucial in geotechnical applications. In MMALE and 
260 CEL methods, the maximum time step size is also restricted by the advection algorithm: the distance of

261 material transport should be less than one element.

\section{Strip footing}

263 The strip footing problem is a well-known benchmark. In this problem, the soil undergoes significant

264 deformation, which challenges the classical Lagrangian methods.

\section{Problem Description}

266 In this problem, large soil deformations are induced by displacement-controlled penetration of a rigid footing. The resulting pressure under the footing can be verified with the analytical solution provided by Hill (1950) using plasticity theory. The footing is initially placed above a container filled with soil. The problem is modeled as plane strain, the lateral boundary nodes of the soil are fixed in the horizontal direction, and the bottom nodes are fixed in the vertical direction. The footing is assumed rigid with smooth (zero friction) sides and a perfectly rough (no slip) base.

Fig. 5 illustrates the initial and boundary conditions of the problem. The strip footing and the soil dimensions are $2 \times 1 \mathrm{~m}$ and $4 \times 4 \mathrm{~m}$, respectively. Only half of the symmetric problem is modeled. The Tresca failure criterion is adopted according to which plastic deformations occur when shear stresses reach the value $c=10 \mathrm{kPa}$, the undrained shear strength of the soil. The Poisson's ratio and the Young's modulus are assigned as $v=0.49$ and $E=2980 \mathrm{kPa}$, respectively. For the ratio of

277 footing base over soil width $=0.5$, the maximum punch pressure for this problem can be calculated

278 from $q_{u l t}=2 c\left(1+\frac{1}{2} \pi\right)($ Hill 1950).

\section{Numerical model consideration}

280 The problem is analyzed using four different methods: Lagrangian, SALE, CEL, and MMALE. The element size in the uniform mesh is $5 \mathrm{~cm}$, with a total number of elements of 3200 . The initial mesh configuration is shown in Fig. 5. The footing in all models is simulated as a rigid body. Frictionless penalty contact between the sides of the footing and the soil is defined.

284 To assess the dependency of results to mesh size, several models with different element sizes were analyzed in another work (Bakroon et al., 2017). The models were solved using SALE method. 
286 Compared to the analytical solution, the optimum mesh size for this problem was reported to be $5 \mathrm{~cm}$.

287 Therefore, $5 \mathrm{~cm}$ mesh size is chosen for all the simulations of this problem.

$288 \quad$ Results

289 The methods are compared based on pressure results and computation time. A Lagrangian model is also developed to highlight the huge mesh distortion. Fig. 6 shows the pressure results under the footing versus penetration depth for Lagrangian, SALE, CEL, and MMALE compared to the analytical solution. By using the Tresca failure criterion, the pressure should reach a constant value after small penetration. Considering the accuracy of results, the Lagrangian and SALE solution differ from the analytical result by approximately $15 \%$ and $10 \%$, respectively. The observed inaccuracy in case of the Lagrangian and SALE can be attributed to several points. The resulting pressure from CEL and MMALE curves follow the same trend as the analytical result, unlike the curves obtained from the Lagrangian and SALE method. It should be noted that initial results included noises which are inevitable in the explicit formulation (Dassault Systèmes, 2016).

299 One may argue that the error is caused due to the element locking (Heisserer et al., 2007). It should be 300 noted that the reduced integration elements are used, which overrules the possibility of element locking. 301 Another possible reason may be the proximity of the boundaries. Comparing the results obtained from the MMALE and CEL and their accurate results, this argument cannot be valid for this problem.

303 Considering the MMALE and CEL results, the distorted element near the corner should be the cause of this problem.

305 The resulting deformation for Lagrangian, SALE, CEL, and MMALE analysis is shown in Fig. 7a.

306 During the Lagrangian solution, the mesh is heavily distorted under the corner of the footing and above.

307 Nevertheless, the simulation continued until the termination time. By using SALE, the overall mesh 308 distortion is alleviated. By using different rezoning methods (e.g., volumetric, equipotential, etc.), 309 different meshes are obtained, but no change in pressure results are observed. In SALE, there are still 310 problems associated with areas around the footing corner where the material encounters significant 311 deformation. These elements are still distorted even with the applied rezoning step. In CEL and 312 MMALE, however, since the material can flow through the mesh, this issue is appropriately addressed. 313 In CEL, the initial mesh is maintained while in MMALE, a new arbitrary mesh is generated. 
314 The instantaneous material velocity field at $0.5 \mathrm{~m}$ penetration depth is plotted in Fig. $7 \mathrm{~b}$. The results of

315 the Lagrangian simulation show a sharp change of the velocity distribution near the lateral boundary of

316 the footing. This is somewhat reduced when using SALE. When using CEL and MMALE, the velocity

317 field is almost uniform in all regions, indicating that the soil particles are moving smoothly

318 counterclockwise from the bottom of the footing to the side and then to the top.

319 In Fig. 8 the effective plastic strain after penetration is shown, which represents the failure pattern of the soil. Despite the identical pressure results shown in Fig. 6, the MMALE provides a clear failure line under the footing. However, CEL underestimates the failure line by providing a discontinued line. This can be attributed to two improvements done by MMALE. First, more elements are present in the failure area. Second, less advection is conducted in MMALE due to remeshing, which avoids loss in accuracy caused by advection.

The performance of each method also is assessed with regard to computation time. The Lagrangian method requires the least computation time among all methods, while the SALE required the most, about three times more than the classical Lagrangian method. The underlying reason is that in SALE two additional steps, remeshing and remapping, are included in the calculation. Another affecting parameter is the distortion of the elements in areas around the corner of the footing since the minimum time step is controlled by those deformed elements. The simple idea behind the implemented smoothing algorithms reduces mesh quality in such non-convex regions instead of improving it, i.e., the smoothing algorithms become unstable. The CEL and MMALE methods solve the problem much faster than SALE because mesh quality is easily maintained. In other words, the minimum time step size did not change

334 significantly during the calculation, unlike SALE. Compared to calculation time obtained from CEL, 335 MMALE is about $40 \%$ faster in spite of an additional rezoning sub-step.

336 The resulting calculation times above for MMALE were based on the optimal set of solution parameters.

337 By using the default settings, a new mesh is generated, and the solution is remapped after each Lagrangian step, which increases calculation time significantly. In many situations, however, the magnitude of deformation obtained after a time increment is small enough to perform several Lagrangian cycles before executing one rezoning and remapping cycle without affecting results considerably. On the other 
341 hand, if the number of Lagrangian cycles before a rezoning and remapping cycle is increased, the mag-

342 nitude of element distortion may reduce the size of the critical time step, which results in more compu-

343 tation cost. Hence, to reach a minimum computation time, an optimum number of Lagrangian cycles

344 should be assigned. This optimum number is problem-dependent, and no predetermination can be 345 made.

346 To optimize the computation cost for the strip footing example, six models are developed where the 347 number of Lagrangian cycles before a remap and rezone cycle varies, ranging from 1 to 30 Lagrangian 348 cycles. To highlight the effect of a number of Lagrangian cycles on calculation time, the mesh size was 349 reduced to $2.5 \mathrm{~cm}$, resulting in 12800 elements. The corresponding calculation times in minutes are 350 drawn in Fig. 9. With the default configuration of MMALE (1 Lagrangian cycle per each rezone and remap cycle), the computation cost is about 70 minutes while assigning 10-20 Lagrangian cycles; it is reduced by $70 \%$. For a large number of Lagrangian cycles, on the other hand, reduction of the critical time step through mesh distortion becomes more pronounced, hence calculation time increases.

354 In this example, by changing the number of Lagrangian cycles, up to $5 \%$ change in pressure results was 355 observed. However, for each problem, the accuracy of the results should be checked since they may be 356 affected by a number of Lagrangian cycles.

357 To investigate this point further, the effect the calculated contact area of the pile with the soil is shown in Fig. 10. In penalty contact method, the contact force is calculated based on the force required to avoid the penetration of the two distinct parts. Generally, this constraint is not adequately maintained and one part "penetrates" or "leaks" inside the other part. In the case of excessive leakage, the contact force will not be accurately computed. To quantitatively investigate this matter, the parameter contact area is used.

362 Theoretically, the value of the contact area should be maintained as of what is calculated at the begin363 ning of the simulation since during the simulation, only the bottom side of the footing is in contact. If 364 this value is increased, it means that leakage has occurred and some of the elements in the second row of the footing has come into contact. In the case of CEL, an increase of $20 \%$ in the contact area is observed. On the other hand, by increasing the number of Lagrangian steps to 50, a significant leakage occurs. Nevertheless, values below this number are providing an acceptable range of leakage. This criterion can be hence used as a limiting factor for a proper number of Lagrangian steps. 
369 In addition, one can see the amount of leakage using a parameter referred to as "flux," which indicates

370 the volume of material passed through the Lagrangian part, in this case, the footing. A high value of

371 flux indicates that a significant volume of material has passed through the Lagrangian part, and there-

372 fore, the errors attributed to leakage are significant. This introduces inaccuracies in the simulation. The 373 computed value of flux is shown in Fig. 11 for both MMALE and CEL. As the simulation continues, 374 the cumulated volume leaked through the Lagrangian footing increases with a faster rate for CEL, which 375 indicates a possibly less accurate result for this method.

376 The effect of mesh size on computation cost for MMALE and CEL is illustrated in Fig. 12 for various 377 cases where the mesh is refined up to 8 times. In addition, the corresponding computation time of ad378 vection for each method is drawn. The computation cost of CEL model is normalized to 1 for each case. 379 he remaining computation times (MMALE, advection in MMALE and CEL) are relatively drawn. In 380 all cases, the MMALE is about $20-40 \%$ faster. However, the trend is not linear, i.e., in the case of one381 fourth of the original size, the computational gain is the least. In all cases of CEL, more than $40 \%$ of 382 the time is spent on advection whereas in case of MMALE it is less than about $30 \%$. The underlying 383 reason is the remeshing step, which reduces the advection calculation by providing a mesh which fol384 lows the material deformation pattern.

385 In the context of the numerical modeling, it is desired to keep the mesh as Lagrangian as possible since 386 the advection procedures introduce errors in the calculation, one of which is the loss of kinetic energy 387 during the advection. Typically, the momentum is preferred over the kinetic energy to be conserved 388 during the advection to maintain the monotonicity of the solution. Maintaining both the momentum and 389 kinetic energy is not possible as it invalidates the monotonicity conditions. This leads to kinetic energy 390 loss during the simulation (Souli and Benson, 2013). To compare the performance of MMALE and 391 CEL regarding this matter, the kinetic energy and the loss of kinetic energy are shown in Fig. 13. The 392 use of remeshing results in a reduction of energy less to almost one-fourth of one calculated by CEL. 393 In the case of kinetic energy curves, the one obtained from CEL is oscillating, which may indicate some 394 instabilities in the method compared to the smooth curve of MMALE. 


\section{Sand column collapse}

396 The collapse of the sand column on a rigid horizontal plane is an experimental test which has various

397 engineering applications such as determining the angle of repose. In the context of geotechnical

398 engineering, this problem can simply represent problems such as a landslide. In such tests, a column of

399 sand is held in a container, and the holding gate is suddenly released, allowing the sand to collapse by

400 its own weight. For further information regarding sand column theories and experiments see the works

401 done by Doyle et al. (2007); Lube et al. (2007); Staron and Hinch (2007).

$402 \quad$ Problem Description

403 An experimental study performed by Lube et al. (2005) has been chosen as a reference model to analyze

404 the robustness of numerical methods. The experimental results of run-out distance and height of the

405 sand column are compared to the obtained numerical values. This problem has been extensively used

406 for performance evaluation of numerical methods such as the work done by Solowski and Sloan (2013).

407 In the experiment, the sand column is placed in a rectangular container. Then, one side of the rectangular

408 container is lifted fast to impose the $2 \mathrm{D}$ flow condition. The initial width of the soil column is $d_{i}=0.0905$

409 m with a height to the width aspect ratio (height to width) of 7. The depth of the test soil in a direction

410 normal to flow is $0.2 \mathrm{~m}$. The friction of the horizontal plane (flowing surface) is equal to internal friction

411 of the sand.

\section{$412 \quad$ Numerical model consideration}

413 Fig. 14 shows the initial configuration of the numerical model. A uniform mesh with an element size of

$41415 \mathrm{~mm}$ is used for the MMALE and CEL simulations. Purely Lagrangian and SALE models were also

415 developed for reasons of comparison. All the models are three-dimensional, defining a slice with one

416 element in a direction normal to the plane. The CEL and MMALE models contain a void region defined

417 to let the soil material flow to these elements after the collapse starts, unlike SALE model where no

418 void elements are needed. Elements with 1-point integration are used, and Mohr-Coulomb is chosen as

419 the material model. Unfortunately, no data regarding the properties of the test sand are reported by Lube

420 et al. (2005). Therefore, the soil properties are assumed as follows, the density, $\rho=1600 \mathrm{~kg} / \mathrm{m}^{3}$, the

421 friction angle, $\phi=33^{\circ}$, the dilatancy angle of $\psi=0$, the cohesion, $c=0.01 \mathrm{kPa}$, the Poisson's ratio, 
$422 v=0.3$, and the elastic modulus, $E=840 \mathrm{kPa}$. The gravity acceleration is $9.806 \mathrm{~m} / \mathrm{s}^{2}$. The left

423 boundary (wall of the container in the experiment) was modeled using a frictionless rigid body part

424 which was removed after the stresses were initialized. The bottom surface was modeled by a rigid body

425 part as well, having tangential penalty friction equal to soil internal friction angle. The run-out distance,

426 as well as the height of the sand column, were measured at different times and compared to numerical

427 results.

$428 \quad$ Results

429 To express the shortcomings of the classical simple based formulations against multi-material based 430 formulations, the problem was also simulated with SALE methods. In this case, the mesh became highly 431 distorted, and the calculation stopped. The mesh clearly tracked the material particles, which can be 432 justified by the concentration of mesh elements as shown in Fig. 15. Due to local rezoning inside the material domain, the mesh quality is to some extent uniform, but elements are severely stretched in the horizontal direction due to the constraints imposed by the material boundary on the remeshing capability. Therefore, after reaching approximately $15 \%$ of the calculation time, the time step size decreased significantly so that the calculation could not be continued. In the case of both CEL and MMALE, simulation continued until the final runout distance of the sand column was reached because of the advection technique, i.e., the material can flow through the mesh. Fig. 16 shows that the remeshing capability of MMALE concentrates the mesh in areas of interest, i.e., where the free surface of the sand is located. The newly generated mesh takes the trend of the material movement and deformation. Hence, the resulting interface is smooth, which is not the case when using the CEL method. The difference in concentration of mesh nodes also affects the final shape of the collapsed sand column, i.e., the final interface of MMALE is curved, whereas the interface of CEL is almost linear. The advantage of MMALE over CEL is also highlighted in Fig. 16, where the volume fraction of sand is plotted. In elements completely filled with sand, the volume fraction equals one, which is represented by blue color. Void elements are drawn in red color, and those elements intersected by the free surface are partially filled with sand, thus have a volume fraction between zero and one.

448 MMALE produces an almost smooth interface, whereas the interface obtained with CEL has a stepped 449 shape and is more diffusive. The diffusion thickness of the interface obtained from CEL is about three 
times more than the one of MMALE. The difference can be attributed to errors caused by remapping.

451 In advection-based remapping methods, only principal directions (normal to element edges) are

452 considered for calculating the advection, neglecting the advection in diagonal directions. Through the MMALE rezoning capability, the element directions are to some extent adjusted to flow directions which results in less remapping errors due to diagonal advection. Moreover, the total advected material volume using an MMALE mesh is usually smaller than for a comparable CEL mesh because the difference between the rezoned mesh and the mesh after the Lagrangian step is reduced.

457 To compare both methods with the experimental measurements, Fig. 17 is plotted, which draws the 458 shape of the sand regime at several times measured during the experiment and calculated by numerical 459 simulations. During the whole simulation, the obtained run-out distance from CEL is underestimated, which becomes more evident at the further stages of the simulation. On the other hand, the MMALE provides a good agreement in the run-out distance with the experiment. Also, at later stages of the simulation, there is a difference in a sand shape calculated by each method. The final sand shape predicted by MMALE is closer to the experimental values than with CEL.

464 By evaluating the kinetic energy loss during advection in Fig. 18, Similar to the strip footing problem, 465 the CEL results in about four times more energy loss than MMALE. This may explain the underestimated run-out distance calculated by CEL which highlights the role of the remeshing in addressing the issues associated with complex and high-speed deformation problems. Nevertheless, the height of the final deformed shape is underestimated, which can be attributed to the employed material model. In any case, the fact that the remeshing step devised in MMALE improved the accuracy, the interface resolution, and the overall deformed shape is highlighted in this problem. In Fig. 19, the location of several material points tracked through the simulation is drawn. In case of

472 ALE, the displacement of any point would be averaged from the displacement of its neighboring mesh nodes in the element containing the point during the Lagrangian step. In the vertical direction, unlike the horizontal direction, both methods predict the same position. The location of the points near the right side of the column changes more notably. The maximum variation between the calculated positions is attributed to point $\mathrm{P} 4$ with almost $30 \mathrm{~cm}$ difference. In this point, the change in both horizontal and vertical direction is extreme and in the diagonal direction of the initially generated 
478 Eulerian mesh. By close observation of the final mesh of the MMALE, it is observed that the elements 479 are arranged in a way to capture the movement of the sand column in this direction. Concerning the fact 480 that a considerable amount of particles undergoes such movements, the MMALE may be a better choice 481 over CEL for this problem.

\section{Soil cutting by blade}

483 Soil cutting tests are conventionally used to design cutting blades. Such problems can also be a good indicator of the ability of a numerical approach to treating material separation, which is similar to the case of pile installation. Different semi-empirical relations are available in the literature for predicting the horizontal and vertical cutting force of the blade (McKyes, 1985). However, these relations are often too simple to deliver acceptable results because the complexity of real soil behavior is not adequately modeled (Onwualu, 1998). Moreover, conducting parametric studies using experiments is costly and time-consuming.

490 Since the material is split during cutting, i.e., new free surfaces are generated, this test is considered as 491 a challenging large deformation problem. In a purely Lagrangian simulation, this would mean that the mesh elements must be separated from each other during the blade progression. Efforts have been made to model such problems using advanced numerical techniques. An application similar to soil cutting by

494 the blade is the penetration of a hollow pile, where the soil is cut by installing the pile.

496 The test consists of a cutting blade with an inclination angle of $45^{\circ}$, which passes through a body of

497 clay, as shown in Fig. 20. The horizontal component of the cutting blade velocity is initialized from 0 up to $0.04 \mathrm{~m} / \mathrm{s}$ in the course of two seconds to avoid instant loading, which induces shock load.

499 Afterward, the velocity is kept constant until the end of the solution. The total simulation time is 24 500 seconds.

\section{$501 \quad$ Numerical model consideration}

502 The soil model used in the simulation is assigned as an elastic-plastic material employing the von-Mises 503 failure criterion which has a density, $\rho=2000 \mathrm{~kg} / \mathrm{m}^{3}$, the cohesion $c=50 \mathrm{kPa}$, the Poisson's ratio $v=0.25$, and the elastic modulus of $E=1000 \mathrm{kPa}$. The parameters are taken from the example in 
505 (Peng et al., 2017) with some modifications. The cutting blade is modeled as a rigid body to minimize

506 the dependency of the model to the blade. The interaction between soil and cutting blade is assigned as

507 a frictionless contact. A uniform mesh size, as shown in Fig. 20 was used with a size of $0.02 \mathrm{~m}$. The

508 model thickness in a perpendicular direction to the plane is $0.05 \mathrm{~m}$. A rather large area of void elements

509 around the elements filled with soil is required to allow the material to flow through the mesh during

510 the cutting process.

$511 \quad$ Results

512 As a first step, the problem has been analyzed using the SALE method. In this method, the mesh deforms

513 significantly, and the solution terminates only after the short amount of time since the elements cannot

514 get "out of the way" of the cutting blade (Fig. 21). Consequently, it is not possible to handle such

515 problems using SALE or Lagrangian methods. By contrast, the results obtained with both CEL and

516 MMALE are reasonable. Fig. 22 shows the material deformation after cutting approximately $0.9 \mathrm{~m}$ of

517 the soil. It can be seen that these methods pose no restrictions concerning the topological changes in the

518 material domain (material separation) as cutting proceeds. The amount of material penetration into

519 cutting blade elements (so-called material leakage) is limited and can be neglected.

520 To verify the performance of both methods, a closed-form analytical solution suggested by McKyes

521 (1985) is presented in eqs. (12)-(14) . $\mathrm{F}_{\mathrm{V}}$ and $\mathrm{F}_{\mathrm{H}}$, therein are the required vertical and horizontal forces, respectively, to cut the soil. The problem is considered as plane strain. In addition, the tool is considered as smooth and rigid (McKyes, 1985).

$$
\begin{gathered}
P=c d \frac{\cot \phi}{\sin \alpha}\left[\left(\frac{1+\sin \phi}{1-\sin \phi}\right) e^{(2 \alpha-\pi) \tan \phi}-1\right]+q d\left(\frac{1+\sin \phi}{1-\sin \phi}\right) \frac{e^{(2 \alpha-\pi) \tan \phi}}{\sin \alpha} \\
F_{H}=P \sin (\alpha+\phi)+c d \cot \alpha \\
F_{V}=P \cos (\alpha+\phi)-c d
\end{gathered}
$$

524 Where $\mathrm{P}$ is the total force per unit width, $\mathrm{c}$ is the cohesion, and $\mathrm{d}$ is the cutting depth. Other parameters are shown in Fig. 23. Using the $c=50 \mathrm{kPa}, d=0.25 \mathrm{~m}, \phi \approx 0^{\circ}, \alpha=45^{\circ}, q=0 \mathrm{kPa}$, and considering the model width of $0.05 \mathrm{~m}$, the forces are calculated as $F_{H}=893 \mathrm{~N}$ and $F_{V}=356 \mathrm{~N}$. Fig. 24 shows the vertical and horizontal forces induced on the cutting blade for both CEL and MMALE, as well as the analytical solution. By assigning the same material model, both methods converge to a 
529 similar value. Compared to the analytical solution, the horizontal and vertical forces from both methods

530 are in good agreement.

531 As a verification measure, internal and kinetic energy were checked. As a rule of thumb, the kinetic 532 energy of the deforming material should not exceed the range of $5 \%$ to $10 \%$ of internal energy during 533 the simulation (Dassault Systèmes, 2016).

534 The internal energy in both MMALE and CEL converge to the same value (Fig. 25); however, in CEL,

535 a sudden jump is observed. Also, a sudden increase is observed in kinetic energy in CEL. Considering

536 the quasi-static condition of the problem, it is unlikely that such sudden variations possibly occur during

537 the simulation. Therefore, it can be argued that MMALE provides more stable and smoother results.

538 Nevertheless, the tolerance for internal to kinetic energy ratio is still in the range of $5 \%$ for both 539 methods.

540 In this problem, the same mesh size is used in both methods. Due to the quasi-static condition applied

541 to the model, the amount of distortion at each time step is limited, which makes it possible to increase

542 the number of a Lagrangian cycle per rezone step in MMALE. The optimized computation cost of

543 MMALE was then almost half of CEL.

\section{Summary and Conclusions}

545 In this research, the effect of the remeshing step in MMALE is evaluated and compared against CEL, a

546 particular case of MMALE where no remeshing is performed. The evaluation is based on the calculation 547 cost optimization, accuracy, and stability. Three large deformation problems were presented and

548 discussed, for which experimental or analytical results are available. By using the remeshing step, the

549 following points were observed in those problems:

550 - Computation cost optimization can be performed by modifying a number of Lagrangian cycles 551 before a rezone and remap cycle. Therefore, in these cases about $20-40 \%$ reduction in calculation 552 time, can be achieved. This is not the case in CEL, as shown in the strip footing and soil cutting 553 problem. 
- Using the MMALE, a better accuracy can be achieved compared to the CEL, for instance in the example of a sand column collapse, the error in the predicted run-out distance calculated by MMALE was $2 \%$ while in the case of CEL it was about $20 \%$.

- Due to the consideration of the material motion, the remeshing step helps to reach a better resolution of the material interface, as shown in the example of a sand column collapse where the diffusion thickness of the interface was three times less than CEL.

- Owing to the remeshing step in MMALE less remap-related errors, including energy loss during advection and material leakage which deteriorate the simulation results, are produced, and better stability is achieved since less volume is transported during the remap step. In the case of the strip footing about $70 \%$ less energy loss and 30\% less leakage was observed.

Finally, it can be concluded that MMALE is suitable, though the highly sophisticated numerical method for applications in geotechnical engineering involving large material deformations and topological changes of the material domain.

The problems discussed here were modeled using simple material constitutive equations. Further investigations are required to assess the performance of more complex material models in conjunction with MMALE. Moreover, the multi-phase simulation, such as the inclusion of pore water pressure has not been performed using MMALE element formulation. Further studies regarding problems with various drainage conditions are needed.

\section{Data Availability}

Data in graphs generated or used during the study are available from the corresponding author by request.

\section{Acknowledgments}

The authors are thankful for the partial financial support obtained from Deutscher Akademischer Austauschdienst (DAAD) with grant number 91561676 and the Elsa-Neumann scholarship of city Berlin (NAFOEG) with grant number T68001.

\section{References}

Aubram, D., Rackwitz, F., Wriggers, P., and Savidis, S. A. (2015). “An ALE method for penetration 

into sand utilizing optimization-based mesh motion." Computers and Geotechnics, Elsevier Ltd, $65,241-249$.

Bakroon, M., Daryaei, R., Aubram, D., and Rackwitz, F. (2017). “Arbitrary Lagrangian-Eulerian Finite Element Formulations Applied to Geotechnical Problems." Numerical Methods in Geotechnics, J. Grabe, ed., BuK! Breitschuh \& Kock GmbH, Hamburg, Germany, 33-44.

Bakroon, M., Daryaei, R., Aubram, D., and Rackwitz, F. (2018). "Multi-Material Arbitrary LagrangianEulerian and Coupled Eulerian-Lagrangian methods for large deformation geotechnical problems." Numerical Methods in Geotechnical Engineering IX: Proceedings of the 9th European Conference on Numerical Methods in Geotechnical Engineering (NUMGE 2018), A. S. Cardoso, J. L. Borges, P. A. Costa, A. T. Gomes, J. C. Marques, and C. S. Vieira, eds., CRC Press, Porto, Portugal, 673-681.

Bakroon, M., Daryaei, R., Aubram, D., and Rackwitz, F. (2019). "Numerical evaluation of buckling in steel pipe piles during vibratory installation." Soil Dynamics and Earthquake Engineering, 122, 327-336.

Bardenhagen, S. G., Brackbill, J. U., and Sulsky, D. (2000). “The material-point method for granular materials." Computer Methods in Applied Mechanics and Engineering, 187(3-4), 529-541.

Bayoumi, H. N., and Gadala, M. S. (2004). "A complete finite element treatment for the fully coupled implicit ALE formulation." Computational Mechanics, 33(6), 435-452.

Belytschko, T., Liu, W. K., and Moran, B. (2000). Nonlinear finite elements for continua and structures. John Wiley, Chichester.

Benson, D. J. (1989). “An efficient, accurate, simple ale method for nonlinear finite element programs." Computer Methods in Applied Mechanics and Engineering, 72(3), 305-350.

Benson, D. J. (1992). "Computational methods in Lagrangian and Eulerian hydrocodes." Computer Methods in Applied Mechanics and Engineering, 99(2-3), 235-394.

Benson, D. J., and Okazawa, S. (2004). "Contact in a multi-material Eulerian finite element formulation." Computer Methods in Applied Mechanics and Engineering, 193(39-41 SPEC. ISS.), 4277-4298.

Berndt, M., Breil, J., Galera, S., Kucharik, M., Maire, P. H., and Shashkov, M. (2011). “Two-step hybrid conservative remapping for multimaterial arbitrary Lagrangian-Eulerian methods." Journal of Computational Physics, Elsevier Inc., 230(17), 6664-6687.

Brackbill, J. U., and Saltzman, J. S. (1982). “Adaptive zoning for singular problems in two dimensions.” Journal of Computational Physics, 46(3), 342-368.

Chazelle, B. (1989). “An optimal algorithm for intersecting three-dimensional convex polyhedra." 30th Annual Symposium on Foundations of Computer Science, 21(4), 671-696.

Chazelle, B. (1994). "Computational geometry: a retrospective." Proceedings of the twenty-sixth annual ACM symposium on Theory of computing - STOC '94, ACM Press, New York, New York, USA, 75-94. 
618 Dassault Systèmes. (2016). "Abaqus: Version 2016 documentation.”

619 Di, Y., Yang, J., and Sato, T. (2007). "An operator-split ALE model for large deformation analysis of geomaterials." International Journal for Numerical and Analytical Methods in Geomechanics, 31(12), 1375-1399.

Donea, J., Giuliani, S., and Halleux, J. P. (1982). “An arbitrary lagrangian-eulerian finite element method for transient dynamic fluid-structure interactions." Computer Methods in Applied Mechanics and Engineering, 33(1-3), 689-723.

Donea, J., Huerta, A., Ponthot, J.-P., and Rodríguez-Ferran, A. (2004). Arbitrary Lagrangian-Eulerian Methods. Encyclopedia of Computational Mechanics, John Wiley \& Sons, Ltd, Chichester, UK.

Doyle, E. E., Huppert, H. E., Lube, G., Mader, H. M., and Sparks, R. S. J. (2007). "Static and flowing regions in granular collapses down channels: Insights from a sedimenting shallow water model." Physics of Fluids, 19(10), 16.

Ghosh, S., and Kikuchi, N. (1991). "An arbitrary Lagrangian-Eulerian finite element method for large deformation analysis of elastic-viscoplastic solids." Computer Methods in Applied Mechanics and Engineering, 86(2), 127-188.

Gingold, R. A., and Monaghan, J. J. (1977). "Smoothed particle hydrodynamics: theory and application to non-spherical stars." Monthly Notices of the Royal Astronomical Society, 181(3), 375-389.

Hallquist, J. (2006). LS-DYNA® theory manual. Livermore Software Technology Corporation, Livermore Software Technology Corporation, California.

Heins, E., and Grabe, J. (2017). "Class-A-prediction of lateral pile deformation with respect to vibratory and impact pile driving." Computers and Geotechnics, 86, 108-119.

Heisserer, U., Hartmann, S., Düster, A., and Yosibash, Z. (2007). "On volumetric locking-free

Hill, R. (1950). The Mathematical Theory of Plasticity. Oxford Classic Texts in the Ph, Clarendon Press.

Hirt, C. W., Amsden, A. A., and Cook, J. L. (1974). “An Arbitrary Lagrangian-Eulerian Computing behaviour of p-version finite elements under finite deformations." Communications in Numerical Methods in Engineering, 24(11), 1019-1032.

648 Hu, Y., and Randolph, M. F. (1998b). "H-adaptive FE analysis of elasto-plastic non-homogeneous soil Method for All Flow Speeds." Journal of Computational Physics, 14, 227-253.

Hu, Y., and Randolph, M. F. (1998a). "A practical numerical approach for large deformation problems in soil." International Journal for Numerical and Analytical Methods in Geomechanics, 22(5), 327-350. with large deformation." Computers and Geotechnics, 23(1-2), 61-83.

Knupp, P., Margolin, L. G., and Shashkov, M. (2002). "Reference Jacobian Optimization-Based Rezone Strategies for Arbitrary Lagrangian Eulerian Methods." Journal of Computational Physics, 176(1), 93-128.

Kucharik, M., and Shashkov, M. (2012). "One-step hybrid remapping algorithm for multi-material arbitrary Lagrangian-Eulerian methods.” Journal of Computational Physics, Elsevier Inc., 231(7), 
Livermore Software Technology Corporation. (2015). LS-DYNA® Keyword user's manual. Livermore, California.

Lube, G., Huppert, H. E., Sparks, R. S. J., and Freundt, A. (2005). "Collapses of two-dimensional granular columns." Physical Review E - Statistical, Nonlinear, and Soft Matter Physics, 72(4), 41301.

661

Lube, G., Huppert, H. E., Sparks, R. S. J., and Freundt, A. (2007). "Static and flowing regions in granular collapses down channels." Physics of Fluids, 19(4), 9.

Margolin, L. G., and Shashkov, M. (2003). "Second-order sign-preserving conservative interpolation

McKyes, E. (1985). "Soil cutting and tillage." Developments in Agricultural Engineering, Developments in agricultural engineering, Elsevier, Amsterdam.

Nazem, M., Sheng, D., Carter, J. P., and Sloan, S. W. (2008). “Arbitrary Lagrangian-Eulerian method for large-strain consolidation problems." International Journal for Numerical and Analytical Methods in Geomechanics, 32(9), 1023-1050.

Noh, W. F. (1964). “CEL: A TIME-DEPENDENT, TWO-SPACE-DIMENSIONAL, COUPLED EULERIAN-LARGIAN CODE." Methods in Computational Physics, B. Alder, S. Fernbach, and M. Rotenberg, eds., Academic Press Inc., New York and London, 393.

Onwualu, A. (1998). "Draught and vertical forces obtained from dynamic soil cutting by plane tillage tools." Soil and Tillage Research, 48(4), 239-253.

Peng, C., Zhou, M., and Wu, W. (2017). "Large Deformation Modeling of Soil-Machine Interaction in Clay." Geomechanics and Geoengineering, Springer, 249-257.

Plaxico, C., Miele, C., Kennedy, J., Simunovic, S., and Zisi, N. (2009). U08 : Finite Element Analysis Crash Model of Tractor-Trailers ( Phase B ). Knoxville, Tennessee.

Qiu, G., Henke, S., and Grabe, J. (2011). “Application of a Coupled Eulerian-Lagrangian approach on 681

Solowski, W. T., and Sloan, S. W. (2013). "Modelling of sand column collapse with material point method." Proceeding of the Third International Symposium on Computational Geomechanics (ComGeo III), S. P. Gyan Pande, ed., Krakow, Poland, 698-705.

Song, Z., Hu, Y., and Randolph, M. F. (2008). "Numerical Simulation of Vertical Pullout of Plate Anchors in Clay." Journal of Geotechnical and Geoenvironmental Engineering, 134(6), 866-875.

Souli, M., and Benson, D. J. (2013). Arbitrary Lagrangian-Eulerian and Fluid-Structure Interaction. Arbitrary Lagrangian-Eulerian and Fluid-Structure Interaction, (M. Souli and D. J. Benson, 
Souli, M., Ouahsine, A., and Lewin, L. (2000). "ALE formulation for fluid-structure interaction problems." Computer Methods in Applied Mechanics and Engineering, 190(5-7), 659-675.

Staron, L., and Hinch, E. J. (2007). "The spreading of a granular mass: Role of grain properties and initial conditions." Granular Matter, 9(3-4), 205-217.

697 Trulio, J., and Trigger, K. R. (1961). Numerical Solution of the One-Dimensional Hydrodynamic Equations in an Arbitrary Time-Dependent Coordinate System. Report UCRL-6522. Livermore, USA.

Wang, D., Bienen, B., Nazem, M., Tian, Y., Zheng, J., Pucker, T., and Randolph, M. F. (2015). “Large deformation finite element analyses in geotechnical engineering." Computers and Geotechnics, Elsevier Ltd, 65(April), 104-114.

Winslow, A. M. (1963). Equipotential zoning of two-dimensional meshes (UCRL-7312). United States.

Winslow, A. M. (1967). "Numerical Solution of the Quasilinear Triangle." Journal of Computational

Tables

\begin{tabular}{|c|c|c|c|}
\hline Application & Criterion & Purpose & $\begin{array}{l}\text { Ref. } \\
\text { No. }\end{array}$ \\
\hline \multirow{10}{*}{$\begin{array}{l}\text { Strip footing } \\
\text { (Section } 0)\end{array}$} & $\begin{array}{l}\text { Induced pressure under the } \\
\text { footing }\end{array}$ & Quantitative comparison with an analytical solution & Fig. 6 \\
\hline & Mesh distortion & $\begin{array}{l}\text { Qualitative comparison of mesh quality } \\
\text { maintenance }\end{array}$ & Fig. 7a \\
\hline & Velocity field in the soil & $\begin{array}{l}\text { Qualitative comparison of the uniformity in the } \\
\text { velocity field }\end{array}$ & Fig. $7 b$ \\
\hline & Effective plastic strain & $\begin{array}{l}\text { Qualitative comparison according to engineering } \\
\text { judgment }\end{array}$ & Fig. 8 \\
\hline & $\begin{array}{l}\text { Number of Lagrangian } \\
\text { cycles in MMALE }\end{array}$ & $\begin{array}{l}\text { Calculation time optimization without deterioration } \\
\text { in the results }\end{array}$ & Fig. 9 \\
\hline & Contact area & Quantitative comparison with the ideal contact area & Fig. 10 \\
\hline & Flux/Leakage & Quantitative comparison with ideal zero leakage & Fig. 11 \\
\hline & Relative computation cost & Evaluation of remeshing and advection effects & Fig. 12 \\
\hline & Mesh density & $\begin{array}{l}\text { Evaluation of the effects concerning the increase in } \\
\text { the calculation time }\end{array}$ & Fig. 12 \\
\hline & Energy loss & Quantitative comparison with zero energy loss & Fig. 13 \\
\hline \multirow{5}{*}{$\begin{array}{l}\text { Sand column } \\
0)\end{array}$} & Mesh distortion & $\begin{array}{l}\text { Qualitative comparison of mesh quality } \\
\text { maintenance }\end{array}$ & Fig. 15 \\
\hline & Interface reconstruction & $\begin{array}{l}\text { Qualitative comparison of improvement in interface } \\
\text { reconstruction }\end{array}$ & Fig. 16 \\
\hline & Run-out distance & $\begin{array}{l}\text { Quantitative comparison with experimental } \\
\text { measurement }\end{array}$ & Fig. 17 \\
\hline & Energy loss & Quantitative comparison with zero energy loss & Fig. 18 \\
\hline & Particle trajectories & $\begin{array}{l}\text { Quantitative comparison of soil particle flow and } \\
\text { evaluation of methods in capturing complex } \\
\text { material movement }\end{array}$ & Fig. 19 \\
\hline
\end{tabular}




\begin{tabular}{|c|c|c|c|}
\hline & Calculation time & $\begin{array}{l}\text { Evaluation of the effect of remeshing in the } \\
\text { reduction of calculation time }\end{array}$ & 0 \\
\hline \multirow{4}{*}{$\begin{array}{l}\text { Soil cutting } \\
\text { (Section 3.3) }\end{array}$} & Mesh distortion & $\begin{array}{l}\text { Qualitative comparison of mesh quality } \\
\text { maintenance }\end{array}$ & $\begin{array}{l}\text { Fig. } 21 \\
\text { Fig. } 22\end{array}$ \\
\hline & $\begin{array}{l}\text { Induced vertical and } \\
\text { horizontal forces on the } \\
\text { blade }\end{array}$ & Quantitative comparison with an analytical solution & Fig. 24 \\
\hline & $\begin{array}{l}\text { Internal and kinetic energy } \\
\text { time histories }\end{array}$ & $\begin{array}{l}\text { Qualitative comparison of the convergence of the } \\
\text { results; verification of the steady state condition }\end{array}$ & Fig. 25 \\
\hline & Calculation time & $\begin{array}{l}\text { Evaluation of the effect of remeshing in the } \\
\text { reduction of calculation time }\end{array}$ & $\begin{array}{l}\text { Section } \\
0\end{array}$ \\
\hline
\end{tabular}

\section{Figures}

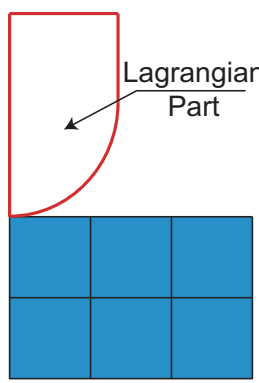

Initial configuration

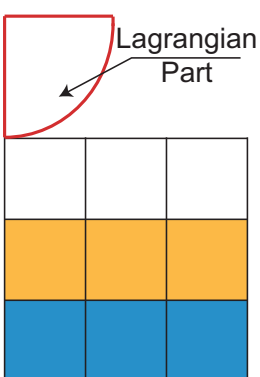

Initial configuration

Material A

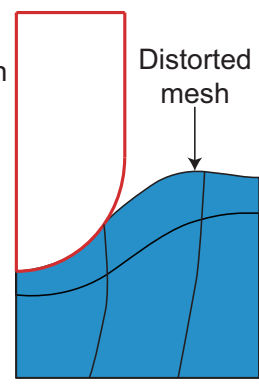

Lagrangian

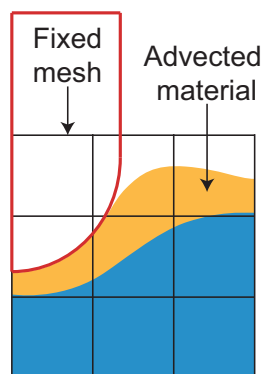

CEL

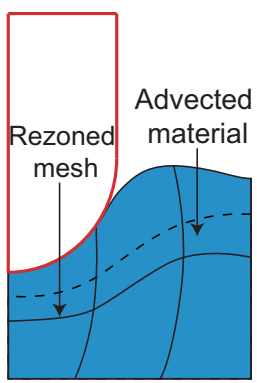

SALE

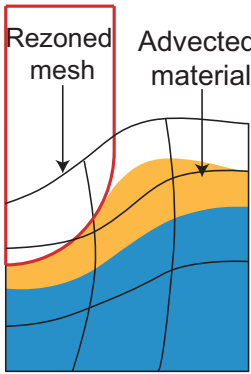

MMALE

Material B $\square$ Lagrangian part

Fig. 1 Schematic diagram of different grid-based approaches comparing the remeshing step effects on grid distortion level.

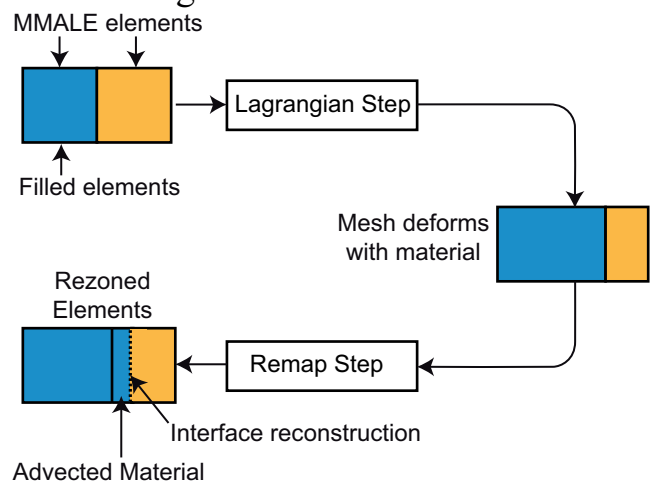

Fig. 2 Flowchart of the operator split scheme applied to the CEL and MMALE calculation steps 

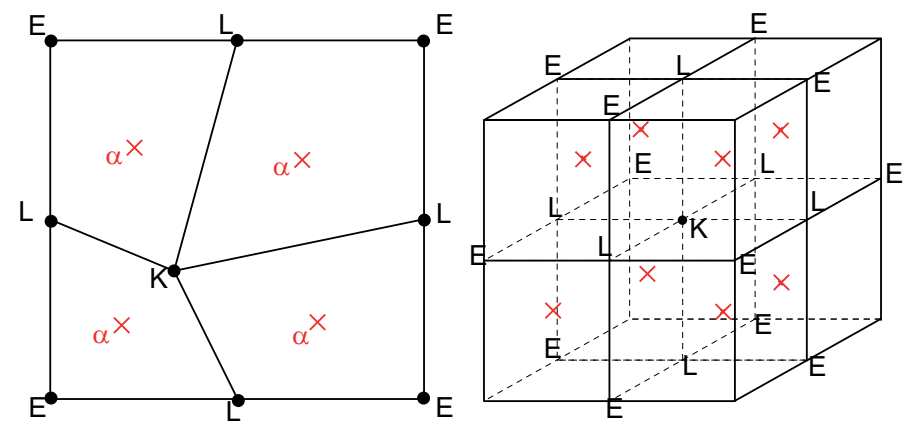

Fig. 3 The initial arrangement of the arbitrary node $\mathrm{K}$ in a grid in $2 \mathrm{D}$ (left) and $3 \mathrm{D}$ (right) used to illustrate the smoothing/remeshing methods described in Eq. (2-(10)

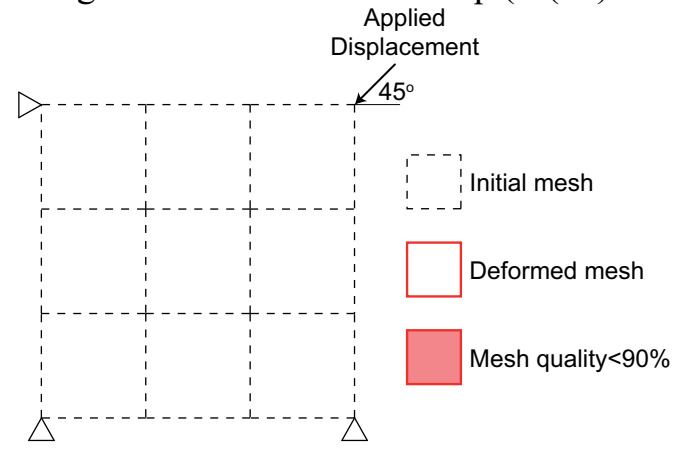

Initial mesh

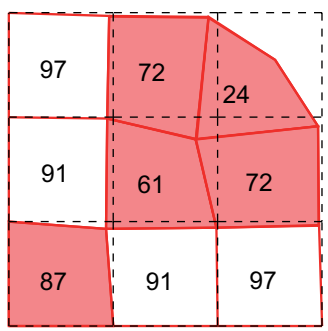

Lagrangian

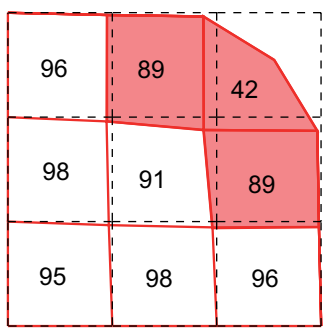

\begin{tabular}{|c|c|c|}
\hline 95 & 90 & 46 \\
1 & 1 \\
\hline 96 & 99 & 90 \\
\hline 96 & 96 & 95 \\
\hline
\end{tabular}

Volume

Laplacian

\begin{tabular}{|c|c|c|}
\hline 96 & 90 & 45 \\
\hline 96 & 97 & 90 \\
\hline 96 & 96 & 96 \\
\hline
\end{tabular}

Equipotential

Fig. 4 Comparison of different smoothing/remeshing algorithms based on the achieved grid quality improvement (the numbers in the squares represents the Jacobian distortion index in percent), the elements colored with red have an element quality less than $90 \%$ 


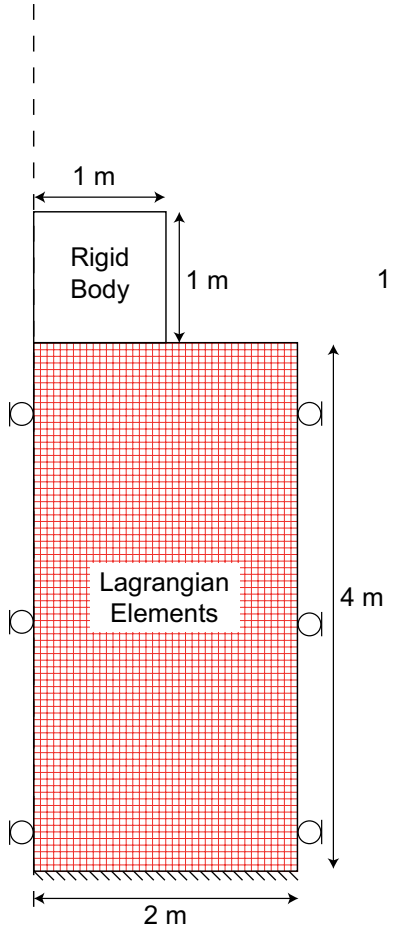

Lagrangian and SALE

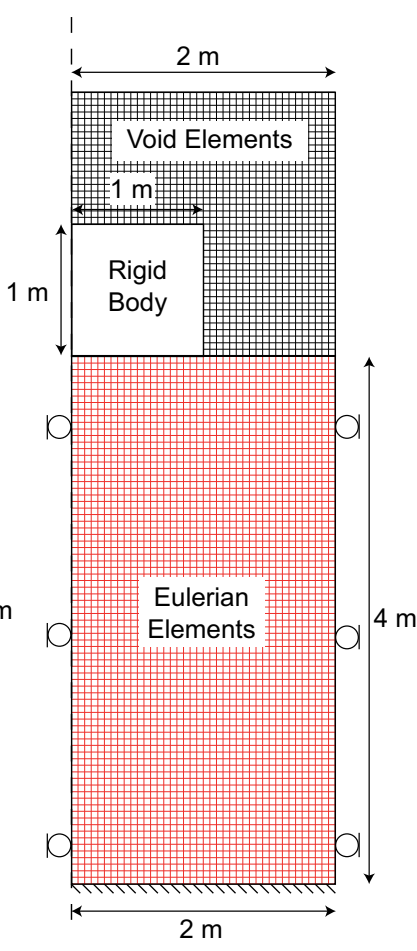

CEL and MMALE

Fig. 5 Numerical mesh configuration of the strip footing problem (Bakroon et al., 2017)

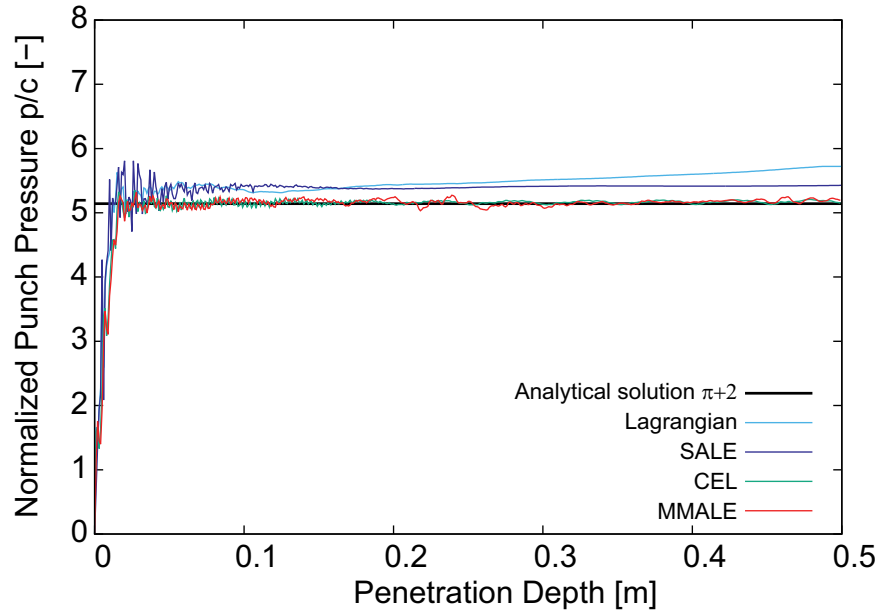

Fig. 6 Comparison of the punch pressure curves obtained from the Lagrangian, SALE, CEL, and MMALE with the analytical solution 


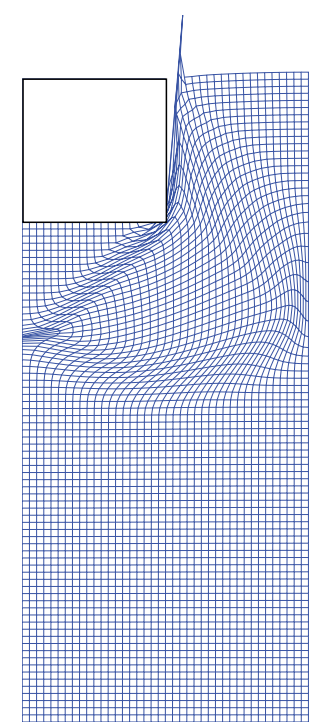

Lagrangian

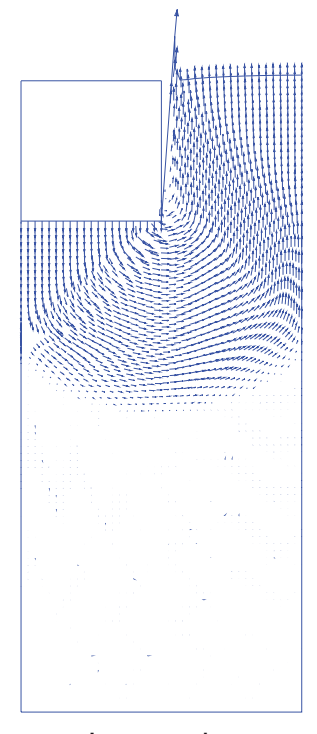

Lagrangian

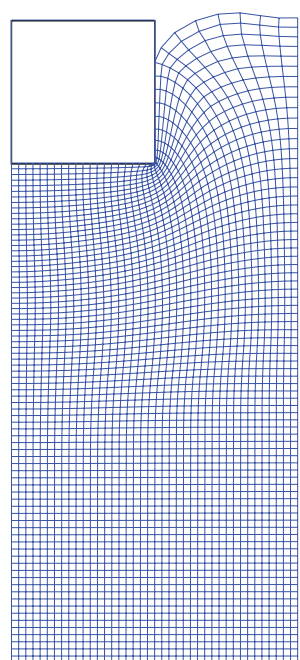

SALE - Volumetric

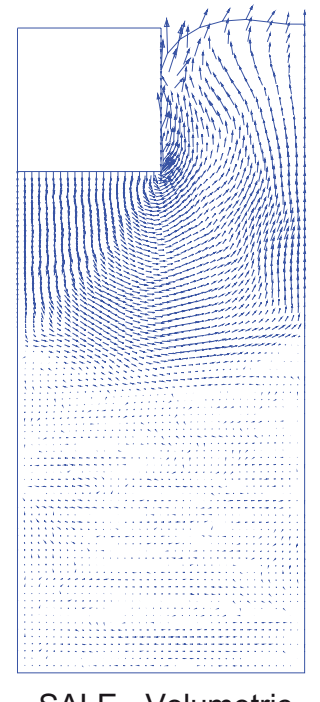

SALE - Volumetric

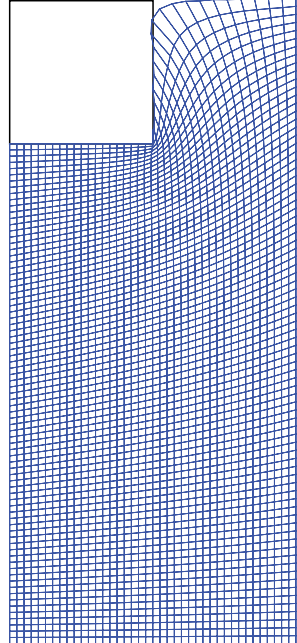

SALE - Equipotential

(a)

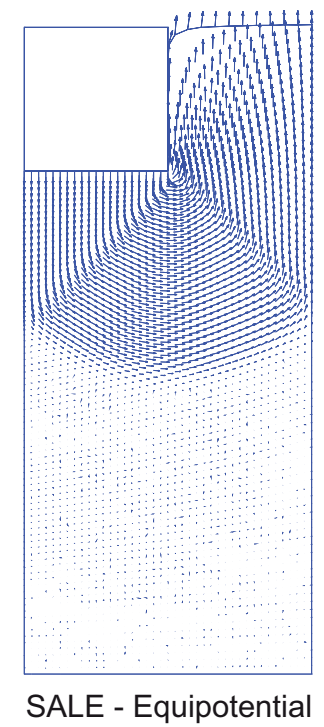

(b)

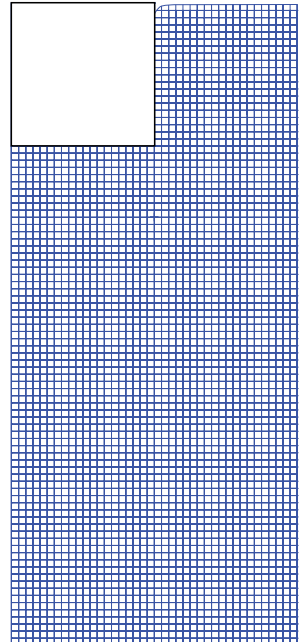

CEL

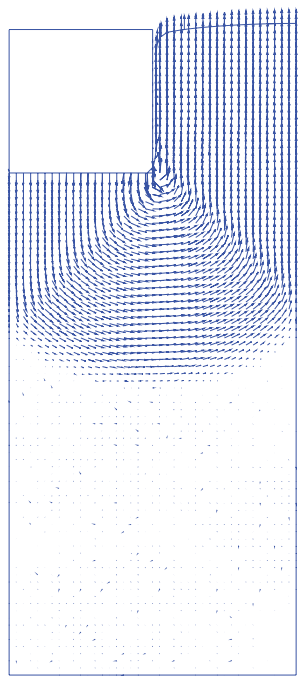

CEL

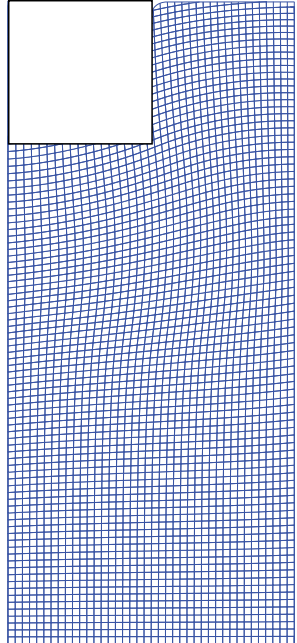

MMALE

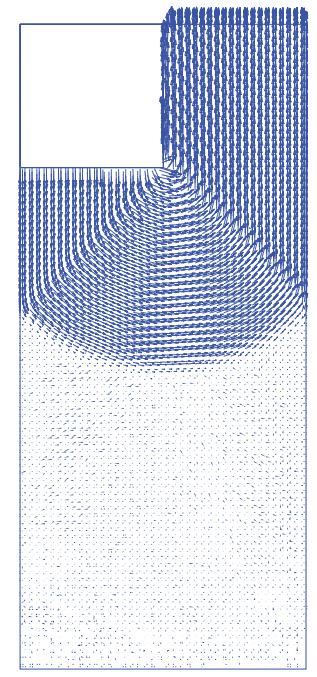

MMALE

Fig. 7 (a) Mesh distortion and (b) velocity field after $0.5 \mathrm{~m}$ of strip footing penetration for different numerical methods 

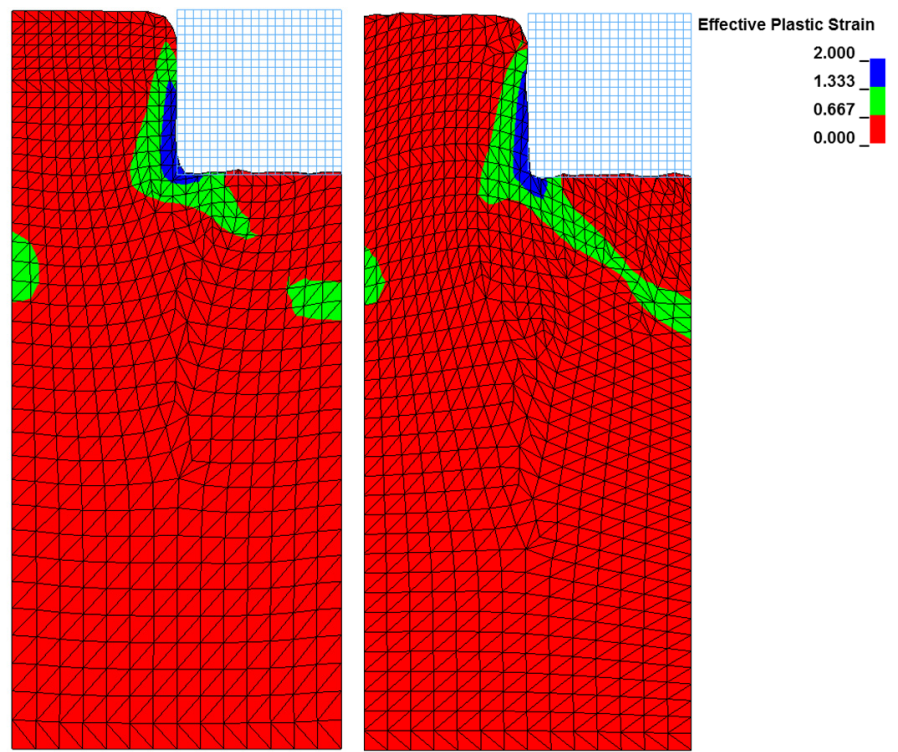

Fig. 8 The effective plastic strain after $0.5 \mathrm{~m}$ penetration for CEL (left) and MMALE (right)

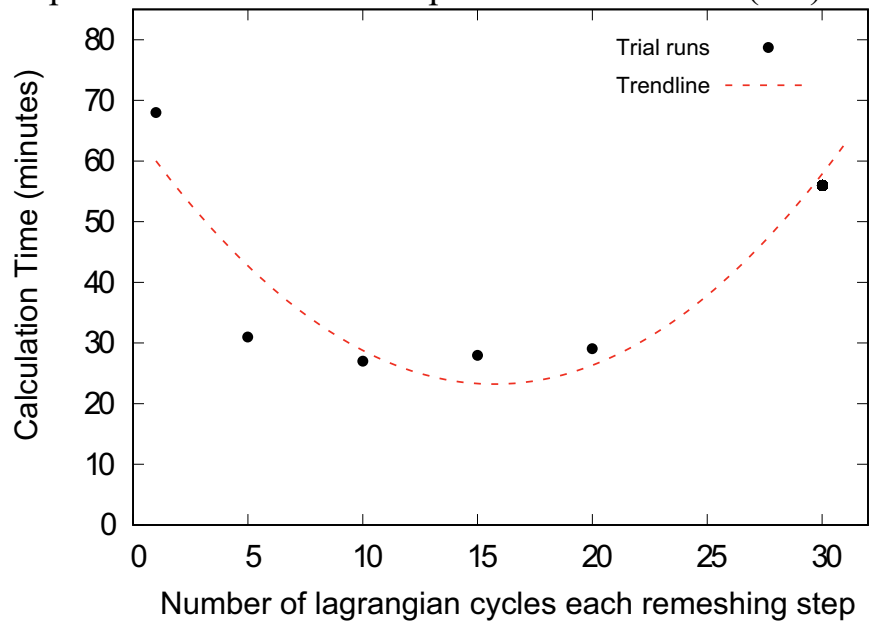

Number of lagrangian cycles each remeshing step

Fig. 9 MMALE time optimization achieved by changing the number of Lagrangian cycles in strip footing problem with $2.5-\mathrm{cm}$ mesh element size

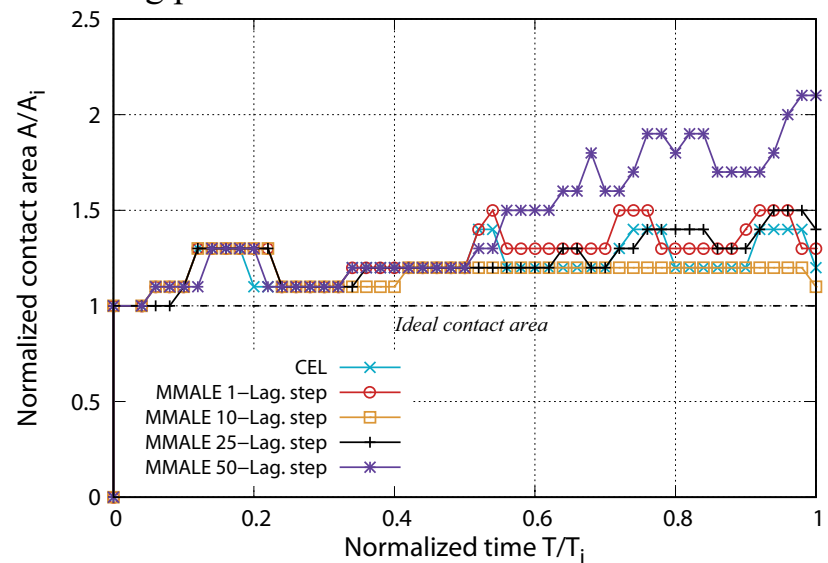

Fig. 10 Change in the normalized contact area during the simulation as a criterion to investigate leakage 
744

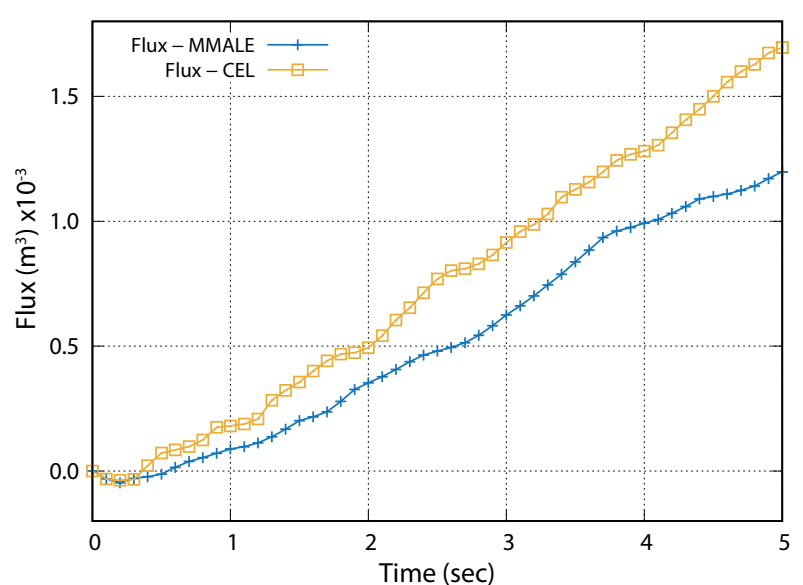

Fig. 11 The amount of material passed through the Lagrangian part (flux/leakage) during the simulation

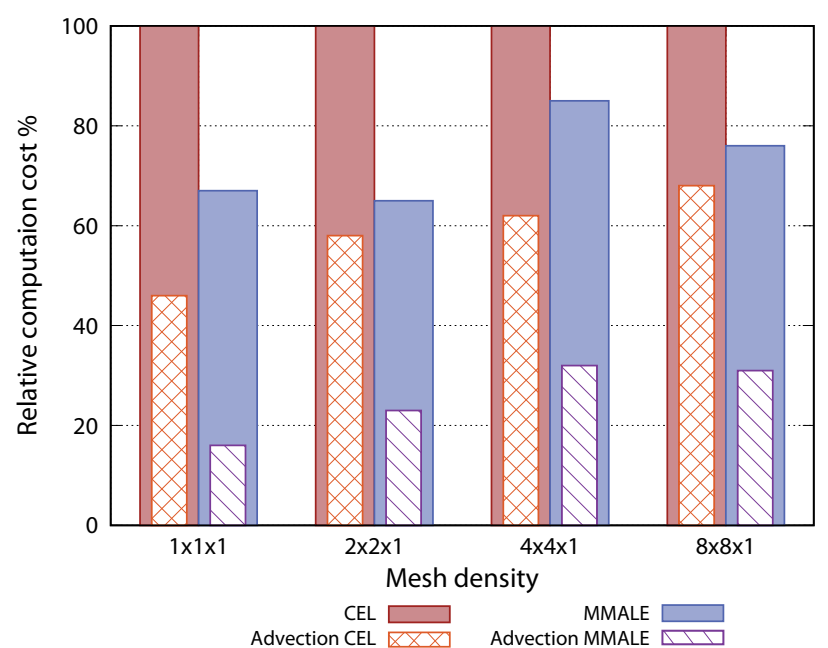

Fig. 12 Relative comparisons of computation costs between CEL and MMALE with their corresponding advection (The results are normalized according to those of CEL for each case)

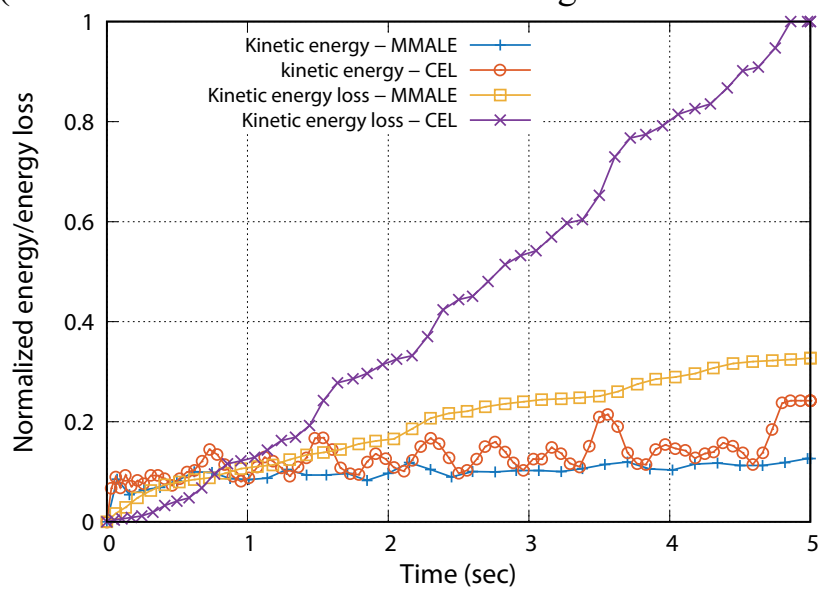

Fig. 13 Normalized kinetic energy and kinetic energy loss during the simulation for MMALE and CEL (the values are normalized with respect to the maximum value of kinetic energy loss curve for CEL) 
754

757

758

761

762

763

764

765

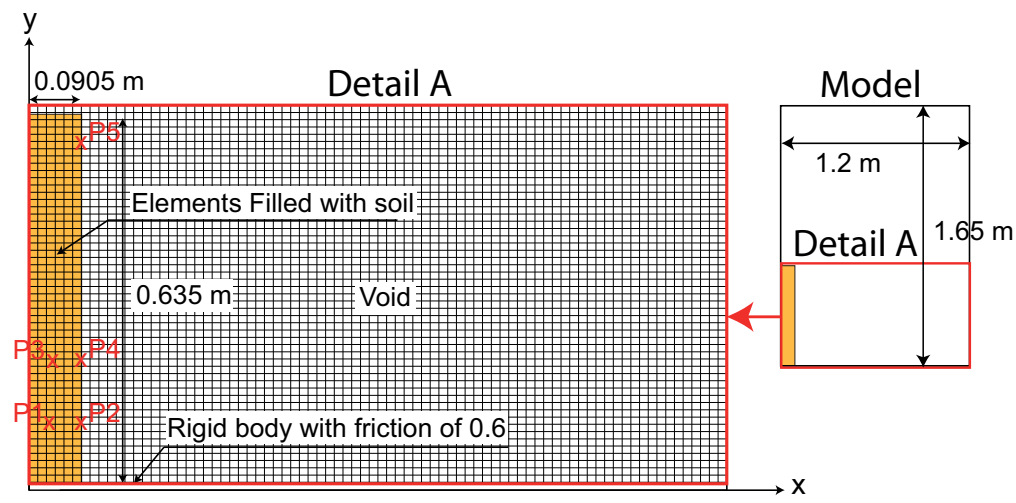

Fig. 14 Initial configuration of the numerical model for the case of CEL and MMALE; the model size is $1.65 \times 1.2 \mathrm{~m}$ but only the mesh of the detail $\mathrm{A}$ is shown

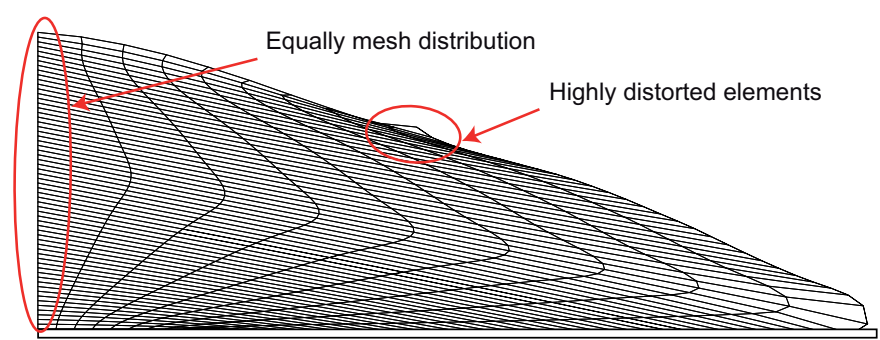

Fig. 15: Mesh deformation for Lagrangian and SALE simulations of sand column collapse

(a)
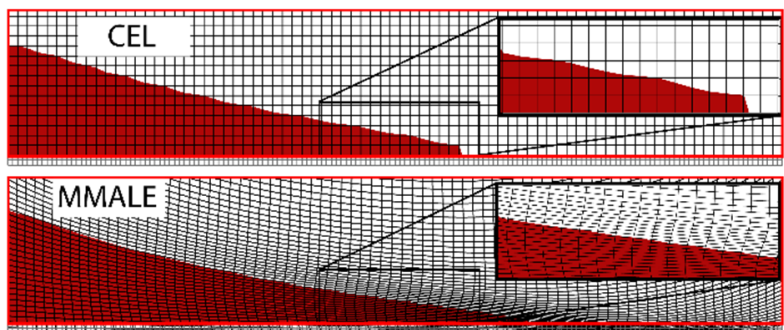

(b)

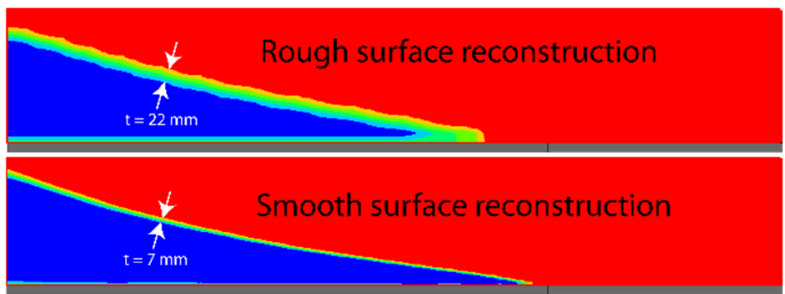

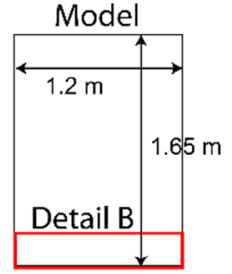

Volume Fraction
1.000
0.833
0.667
$0.500_{-}$
$0.333_{\text {_ }}$
0.167
0.000

Fig. 16 (a) Final shape of the flowed soil as well as the mesh distortion in the sand column collapse for CEL (top) and MMALE (bottom), (b) Soil interface reconstruction in CEL (top) and MMALE (bottom), the contours represent the volume fraction of the soil in the elements; the results correspond to the detail $\mathrm{B}$ and not the whole model 

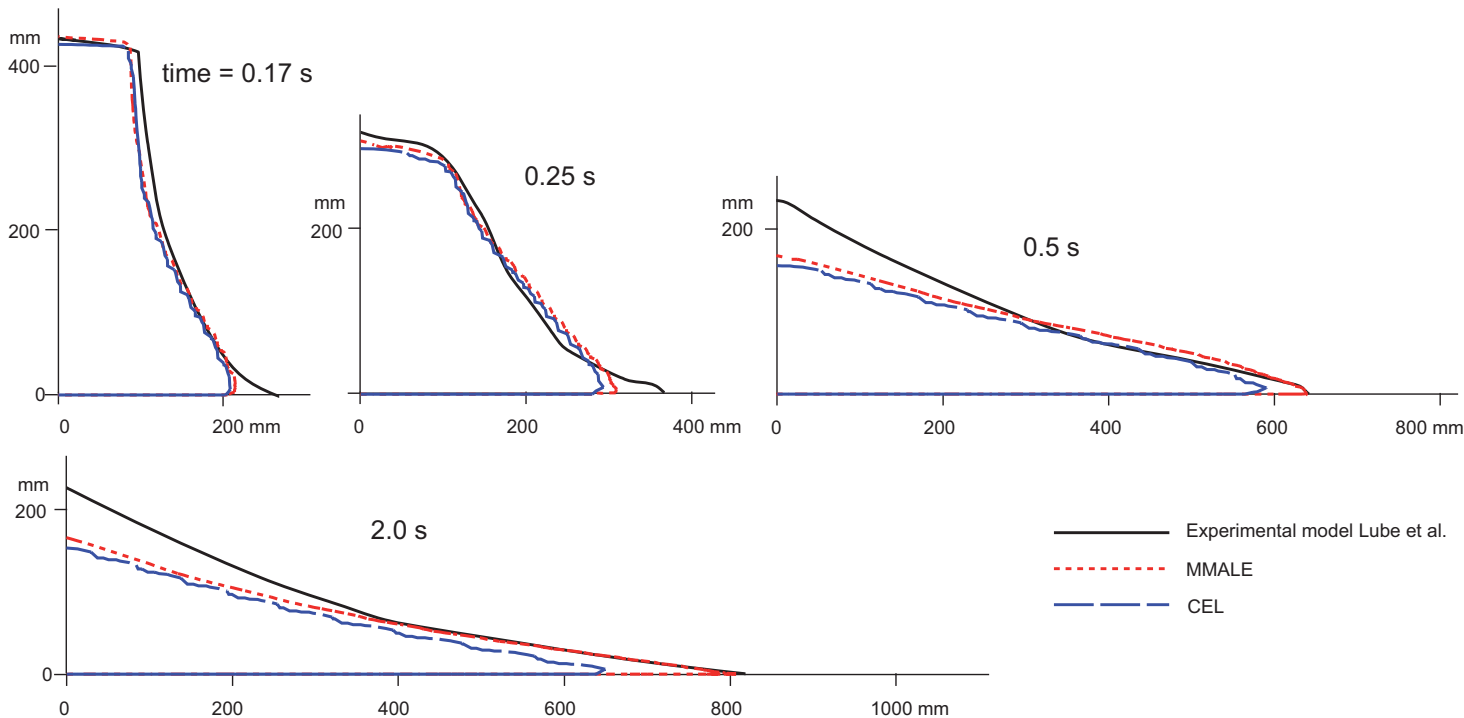

200

400

$800 \mathrm{~mm}$

Fig. 17 Comparison of the run-out distance obtained from the numerical models with the experimental measurements in the sand column collapse problem
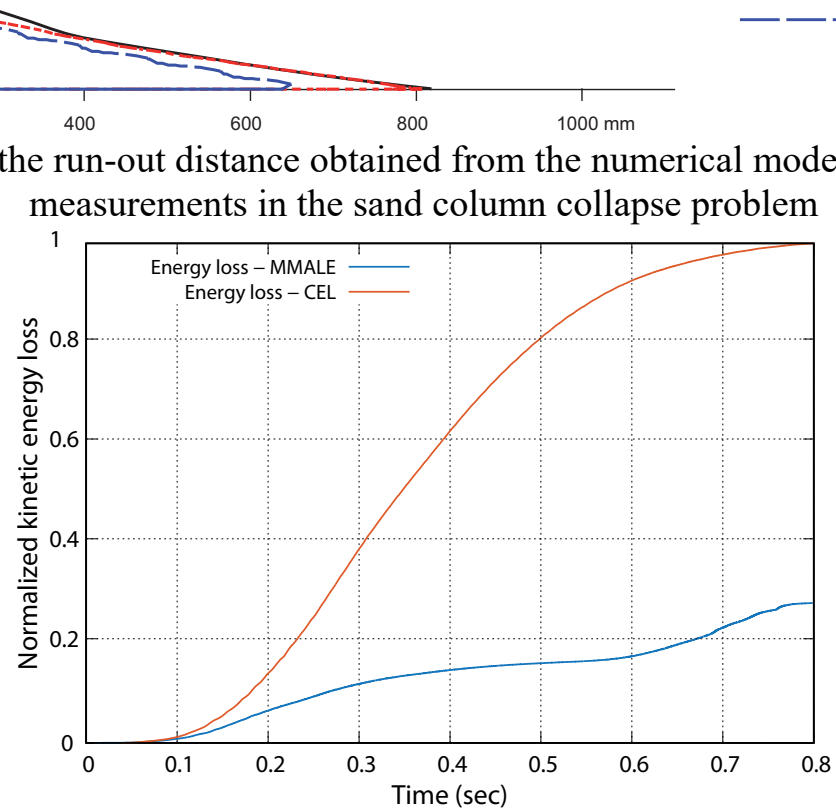

Fig. 18 Comparison of the normalized kinetic energy loss during advection for the sand column problem (the values are normalized with respect to the maximum value of CEL curve)

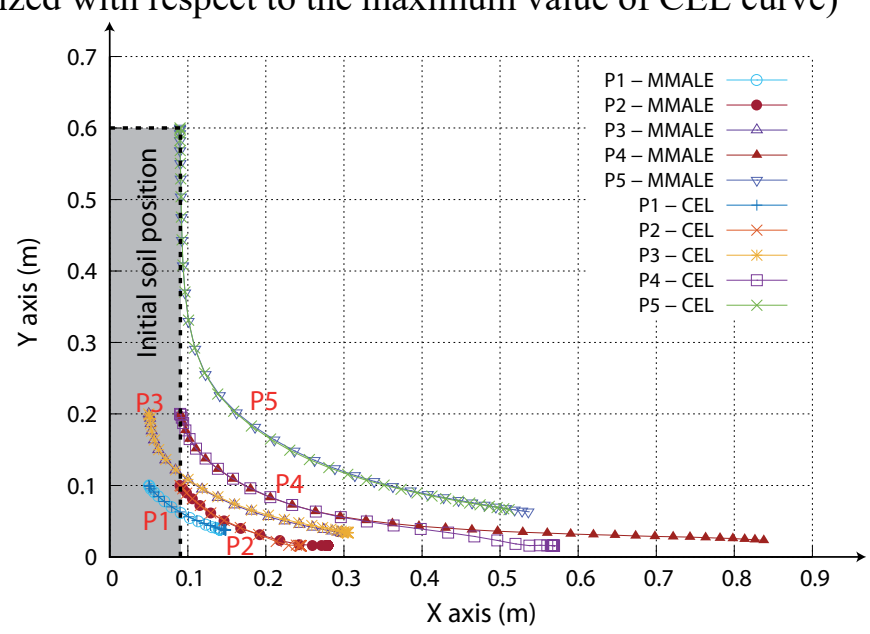

(a) 
774

775

776

777

778

779

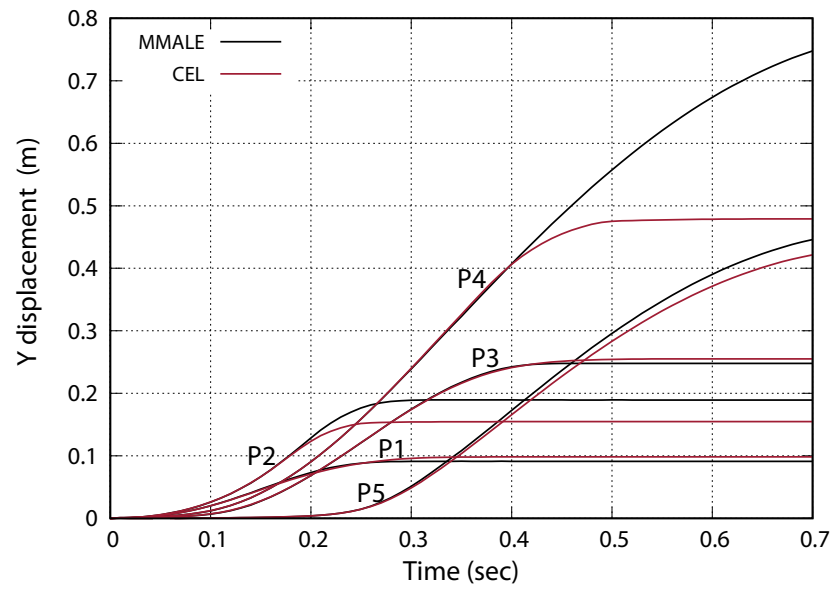

(b)

Fig. 19 (a) soil particle trajectory, (b) Comparison of the displacement between several particles obtained from CEL and MMALE

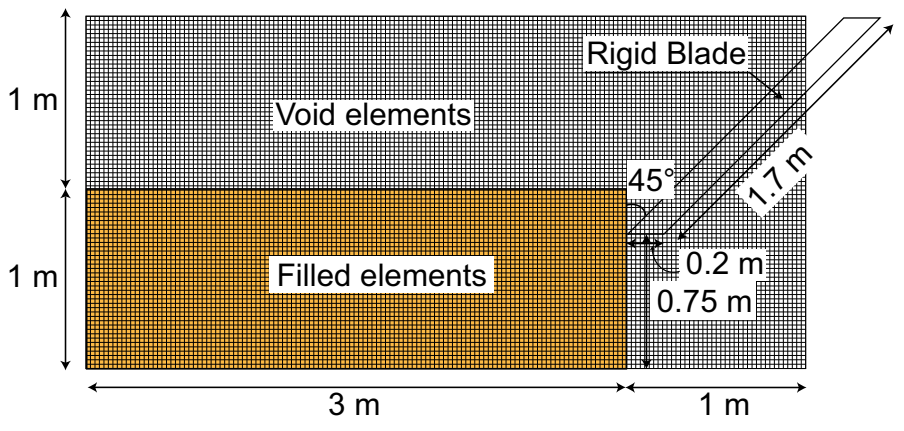

Fig. 20 Schematic view of the soil cutting problem

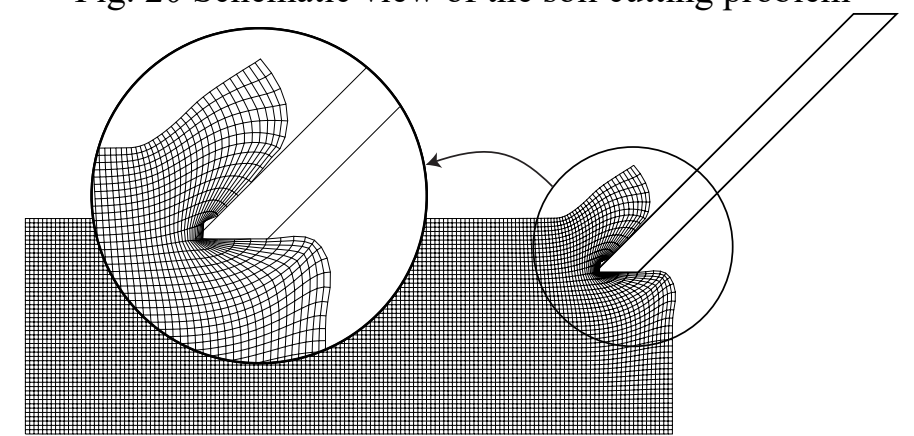

Fig. 21 Mesh distortion during the soil cutting using the SALE method 
782

785

786

787
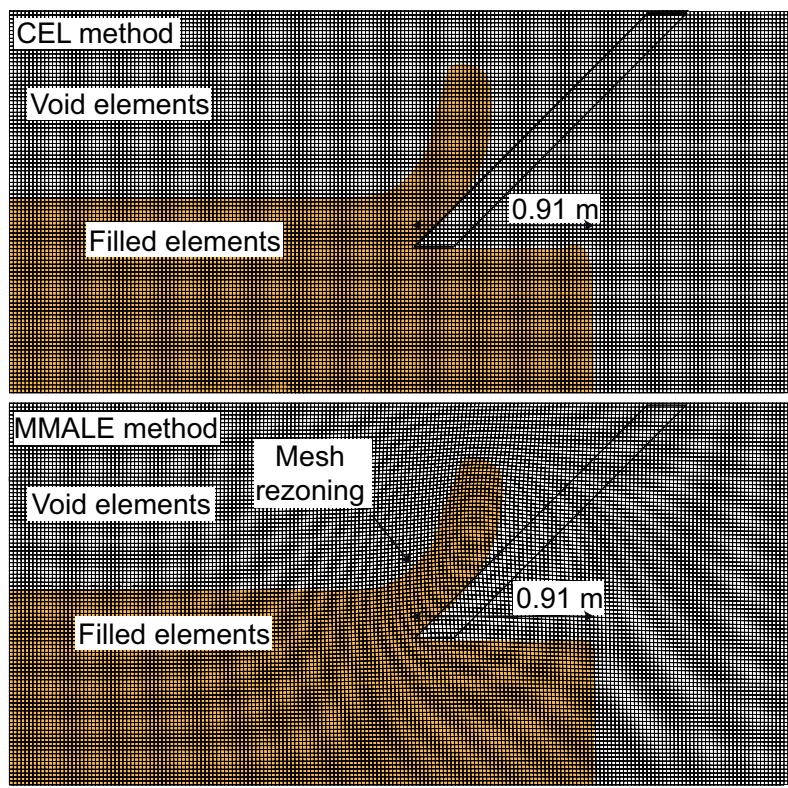

Fig. 22 Mesh distortion and soil deformation using CEL (above) and MMALE (below) methods in the soil cutting problem

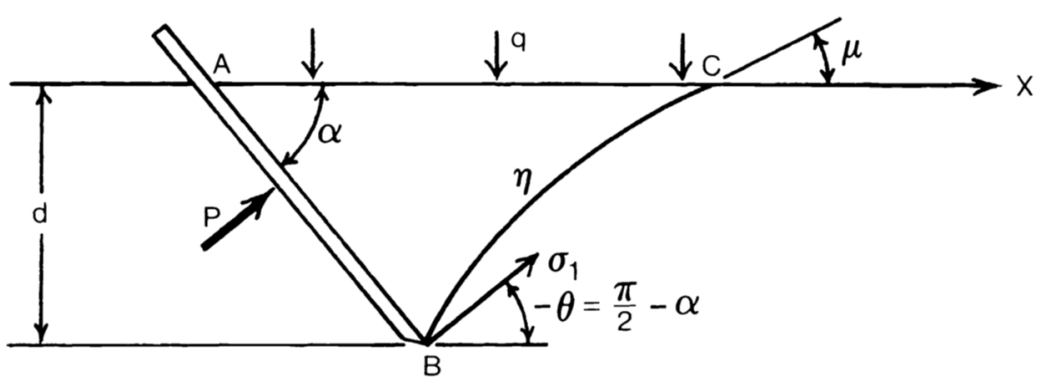

Fig. 23 Schematic of the assumed conditions in the soil cutting problem for deriving an analytical

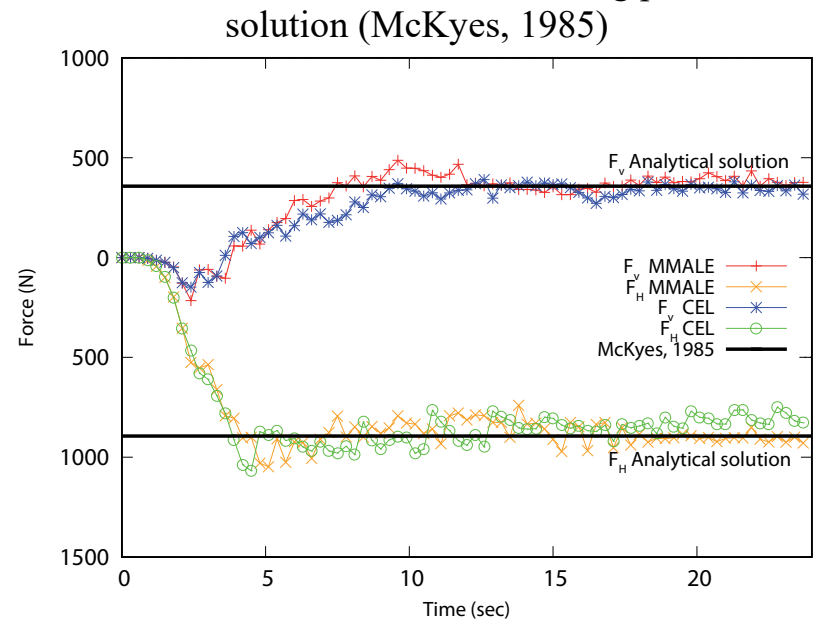

Fig. 24 Comparison of the induced horizontal and vertical forces on the blade obtained from

MMALE and CEL methods with the analytical solution in the soil cutting problem 


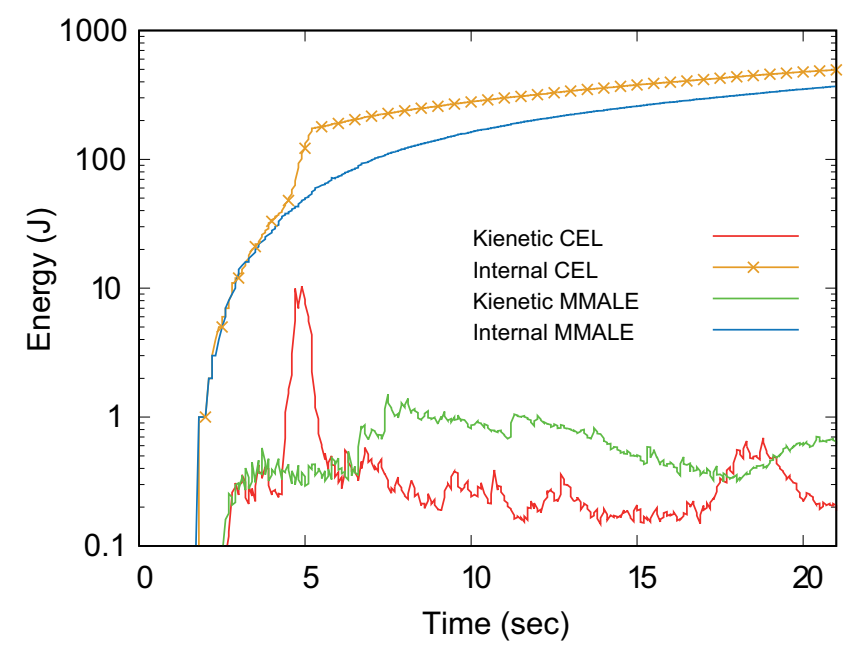

Fig. 25 Comparison of the internal and kinetic energy curves of the soil cutting problem

\section{List of figure captions}

Fig. 1 Schematic diagram of different grid-based approaches comparing the remeshing step effects on grid distortion level.

Fig. 2 Flowchart of the operator split scheme applied to the CEL and MMALE calculation steps Fig. 3 The initial arrangement of the arbitrary node K in a grid in 2D (left) and 3D (right) used to illustrate the smoothing/remeshing methods described in Eq. (2-(10)

Fig. 4 Comparison of different smoothing/remeshing algorithms based on the achieved grid quality improvement (the numbers in the squares represents the Jacobian distortion index in percent), the elements colored with red have an element quality less than $90 \%$

Fig. 5 Numerical mesh configuration of the strip footing problem Bakroon et al. (2017)

Fig. 6 Comparison of the punch pressure curves obtained from the Lagrangian, SALE, CEL, and MMALE with the analytical solution

Fig. 7 (a) Mesh distortion and (b) velocity field after $0.5 \mathrm{~m}$ of strip footing penetration for different numerical methods

Fig. 8 The effective plastic strain after $0.5 \mathrm{~m}$ penetration for CEL (left) and MMALE (right)

Fig. 9 MMALE time optimization achieved by changing the number of Lagrangian cycles in strip footing problem with 2.5 -cm mesh element size 
811 Fig. 10 Change in the normalized contact area during the simulation as a criterion to investigate

812 leakage

813 Fig. 11 The amount of material passed through the Lagrangian part (flux/leakage) during the

814 simulation

815 Fig. 12 Relative comparisons of computation costs between CEL and MMALE with their 816 corresponding advection (The results are normalized according to those of CEL for each case)

817 Fig. 13 Normalized kinetic energy and kinetic energy loss during the simulation for MMALE

818 and CEL (the values are normalized with respect to the maximum value of kinetic energy loss

819 curve for CEL)

820 Fig. 14 Initial configuration of the numerical model for the case of CEL and MMALE; the

821 model size is $1.65 \times 1.2 \mathrm{~m}$ but only the mesh of the detail A is shown

822 Fig. 15: Mesh deformation for Lagrangian and SALE simulations of sand column collapse

823 Fig. 16 (a) Final shape of the flowed soil as well as the mesh distortion in the sand column

824 collapse for CEL (top) and MMALE (bottom), (b) Soil interface reconstruction in CEL (top)

825 and MMALE (bottom), the contours represent the volume fraction of the soil in the elements;

826 the results correspond to the detail B and not the whole model

827 Fig. 17 Comparison of the run-out distance obtained from the numerical models with the

828 experimental measurements in the sand column collapse problem

829 Fig. 18 Comparison of the normalized kinetic energy loss during advection for the sand column

830 problem (the values are normalized with respect to the maximum value of CEL curve)

831 Fig. 19 (a) soil particle trajectory, (b) Comparison of the displacement between several particles 832 obtained from CEL and MMALE

833 Fig. 20 Schematic view of the soil cutting problem

834 Fig. 21 Mesh distortion during the soil cutting using the SALE method 
835 Fig. 22 Mesh distortion and soil deformation using CEL (above) and MMALE (below)

836 methods in the soil cutting problem

837 Fig. 23 Schematic of the assumed conditions in the soil cutting problem for deriving an

838 analytical solution McKyes (1985)

839 Fig. 24 Comparison of the induced horizontal and vertical forces on the blade obtained from

840 MMALE and CEL methods with the analytical solution in the soil cutting problem

841 Fig. 25 Comparison of the internal and kinetic energy curves of the soil cutting problem

842 